\title{
QUANTUM DOT DEPOSITION INTO PDMS AND APPLICATION ONTO A SOLAR CELL
}

\author{
A thesis Presented to the Faculty of \\ California Polytechnic State University, San Luis Obispo \\ In Partial Fulfillment of the Requirements for the Degree of: \\ Master of Science in Engineering: Materials
}

by

Christopher M. Botros

December 2012 
(C) 2012

Christopher M, Botros

ALL RIGHTS RESERVED 


\section{COMMITTEE MEMBERSHIP}

TITLE: Quantum Dot Deposition into PDMS and Application onto a Solar Cell

AUTHOR: Christopher Botros

DATE SUBMITTED: 12/12/2012

COMMITTEE CHAIR: Richard Savage, PhD

COMMITTEE MEMEBER: Lily Laiho, PhD

COMMITTEE MEMBER: Dan Walsh, PhD 


\section{Abstract \\ Christopher M. Botros \\ QUANTUM DOT DEPOSITION INTO PDMS AND APPLICATION ONTO A SOLAR CELL}

Research to increase the efficiency of conventional solar cells is constantly underway. The goal of this work is to increase the efficiency of conventional solar cells by incorporating quantum dot (QD) nanoparticles in the absorption mechanism. The strategy is to have the QDs absorb UV and fluoresce photons in the visible region that are more readily absorbed by the cells. The outcome is that the cells have more visible photons to absorb and have increased power output. The QDs, having a CdSe core and a ZnS shell, were applied to the solar cells as follows. First, the QDs were synthesized in an octadecene solution, then they were removed from the solution and finally they were dried and deposited into polydimethylsiloxane (PDMS) and the PDMS/QD composite is allowed to cure. The cured sample is applied to a silicon solar panel. The panel with the PDMS/QD application outputs $2.5 \%$ more power than the one without, under identical illumination by a tungsten halogen lamp, using QDs that fluoresce in the orange region. This work demonstrates the feasibility of incorporating QDs to increase the efficiency of conventional solar cells. Because the solar cells absorb better in the red region, future effort will be to use QDs that fluoresce in that region to further boost cell output. 


\section{Acknowledgments}

I would like to thank Dr. Savage for all of his help, support and inspiration. You have made materials engineering a much more fascinating subject. I also thank Dr. Laiho for her willingness to help, contributions and ideas. Dr. Walsh's support and belief in the student in the midst of difficulty are irreplaceable, I thank him for all of his help and support. I would also like to thank MST for all of their time and energy to make the field of materials all the more interesting. 


\section{Table of Contents}

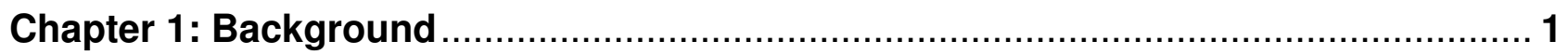

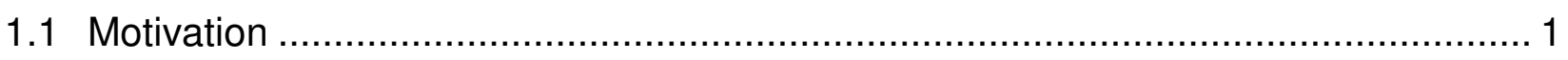

1.2 Quantum Dots and their Applications ……................................................... 3

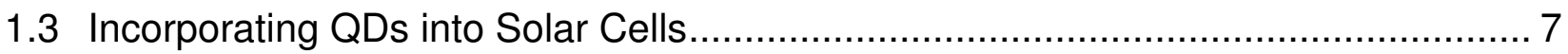

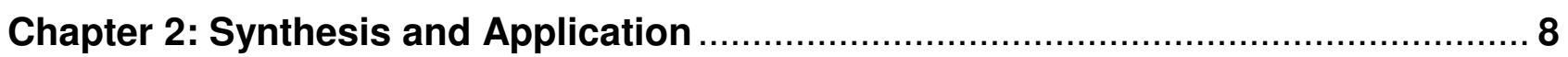

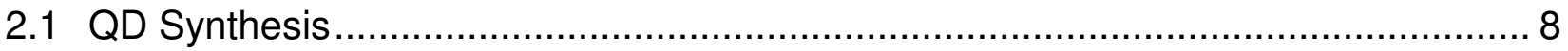

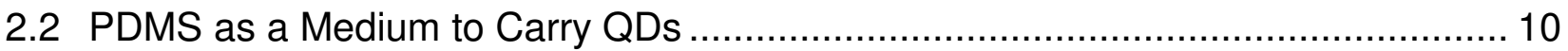

2.3 Necessary QD state for PDMS Deposition ....................................................... 11

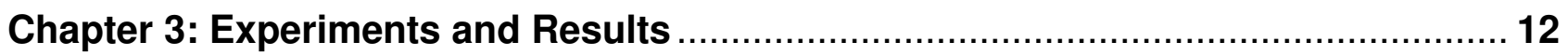

3.1 QD Extraction from Octadecene .............................................................. 12

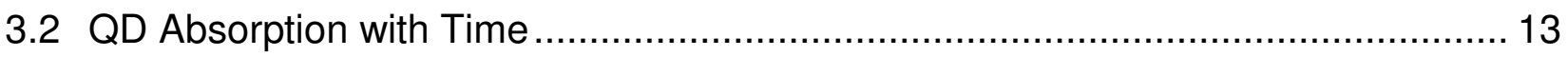

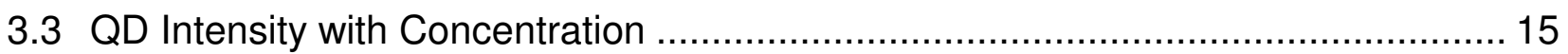

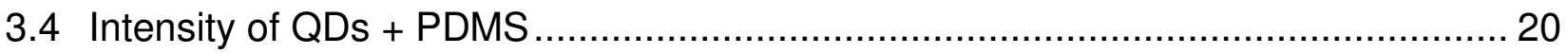

3.5 Solar Cell Efficiency when Applied with QDs + PDMS ......................................... 23

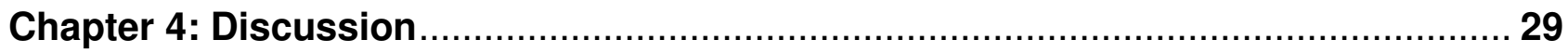

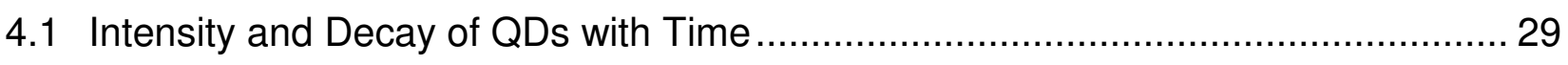

4.2 Spectrometer Variation and Indications of Intensity ............................................ 30

4.3 Accuracy of the Intensity with Concentration Relation ......................................... 33

4.4 Losses of QD Intensity upon Deposition in PDMS ............................................. 35

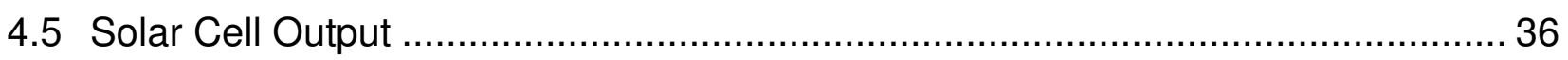

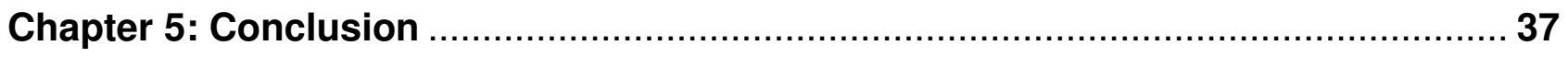

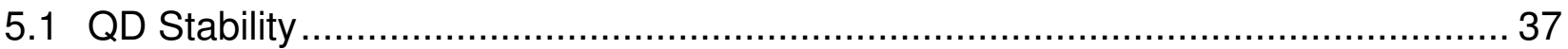

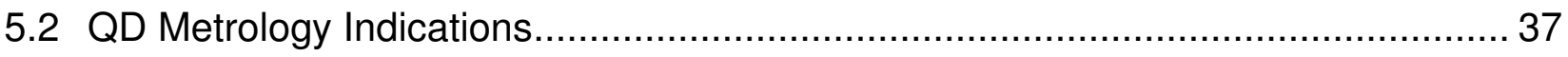

5.3 Solar Cell Efficiency when Applied with QDs .................................................... 38

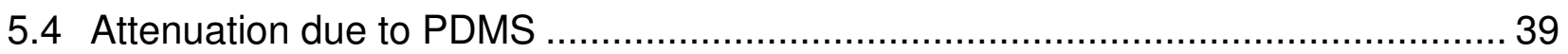

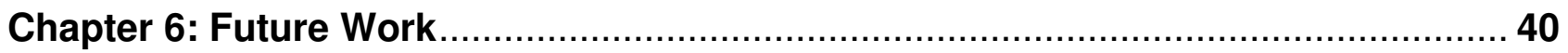

6.1 Alternate Materials as Carrying Mediums …....................................................... 40 


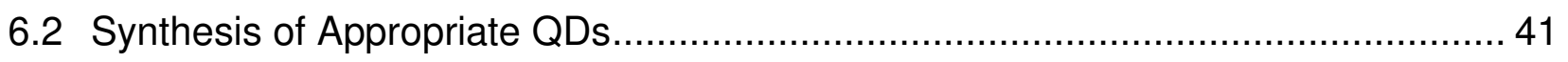

6.3 Solar Cells with Different Band Gaps............................................................. 42

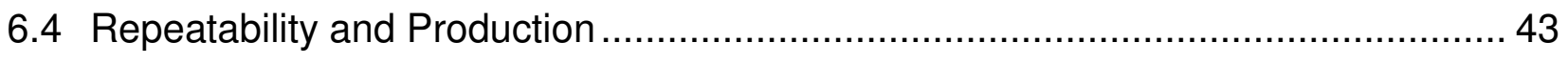

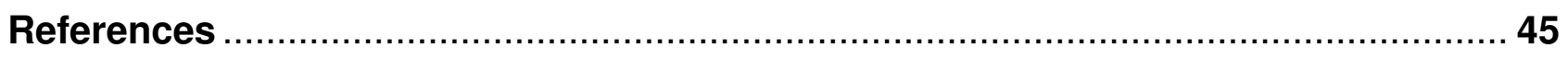




\section{List of Tables}

Table I - Different Solar cells and Their Corresponding Band Gaps …........................ 8

Table II - Removal Times and Quantum Dot Size ................................................... 9

Table III - A comparison of QD intensity when deposited in either PDMS or Toluene ... 22

Table IV - Solar Cell Max Power Bare and Applied with PDMS ................................... 26

Table V - Solar Cell Max Power Applied with PDMS and PDMS + QDs ....................... 27

Table VI - Solar Cell Max Power Bare and Applied with PDMS + QDs ......................... 28

Table VII - Collected Intensity Measurements .......................................................... 31 


\section{List of Figures}

Figure 1 - Electron Relaxation and Excitation.................................................... 2

Figure 2 - Quantum Dot Size and Fluorescing Wavelengths........................................ 4

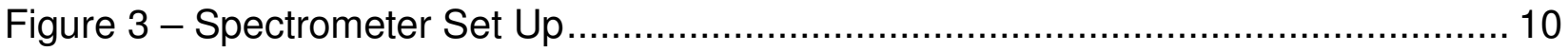

Figure 4 - QD intensity over a 3 Month Period ........................................................... 14

Figure 5 - Intensity of Fluorescein over Different Concentrations................................. 16

Figure 6 - Intensity of Fluorescein over Different Concentrations in the Linear Region.. 17

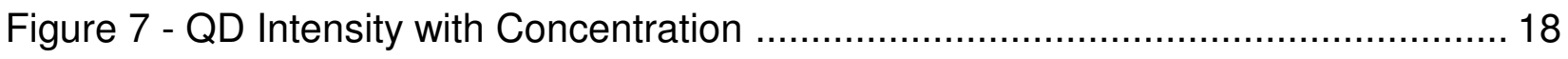

Figure 8 - QD Intensity with Concentration in the Linear Region.................................. 20

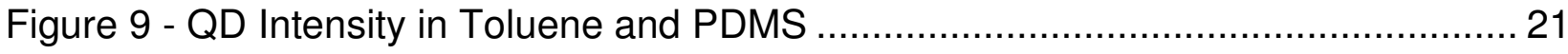

Figure 10 - QD Intensity in Toluene and PDMS in the Linear Region........................... 23

Figure 11 - Solar Cell Power with Various Applications .............................................. 25

Figure 12 - Intensity of Sample over a Single Day ................................................... 30

Figure 13 - Intensity Distribution of QDs ............................................................... 33 


\section{Equations}

Equation 1 - Energy - Wavelength Relation ...................................................... 24 


\section{Chapter 1 - Background}

\subsection{Motivation}

Solar cells are becoming an increasingly important energy source; work is constantly being done to increase their efficiency. Solar efficiency is limited due to the quantum efficiency and the thermodynamic efficiency limit of the cell.

Quantum efficiency refers to photons that are absorbed by the solar cell to produce electron-hole pairs. The electron-hole pair can either produce a carrier that will carry an electric charge or the carrier will recombine resulting in no current for the solar cell. Quantum efficiency is the ratio of the carriers that produce an electric charge to those that recombine [1].

Thermodynamic efficiency limits the power output of a solar cell due to the band gap and the Carnot limit associated with the solar cell. Photons with energy below the band gap will not produce an electron-hole pair (exciton) so the energy is not converted to a power output. Photons with energy greater than the band gap will cause an electron to be excited to the conduction band and then relax to the valence band, with the excess energy being converted to heat [2]. With increasing heat, the solar cell will suffer from heat related energy losses due to increases in resistance. 
For the reasons just stated, solar cell performance increases when irradiated with photons within the region most readily absorbed by the cell's band gap. Quantum dots (QDs) can absorb photons with energy greater than the band gap and fluoresce at an energy specific to the band gap, thus providing the solar cells with photons only within a given cell's particular band gap. In doing this, the solar cell is much cooler since much of the excess energy from photons of greater energy than the band gap no longer produces heat.

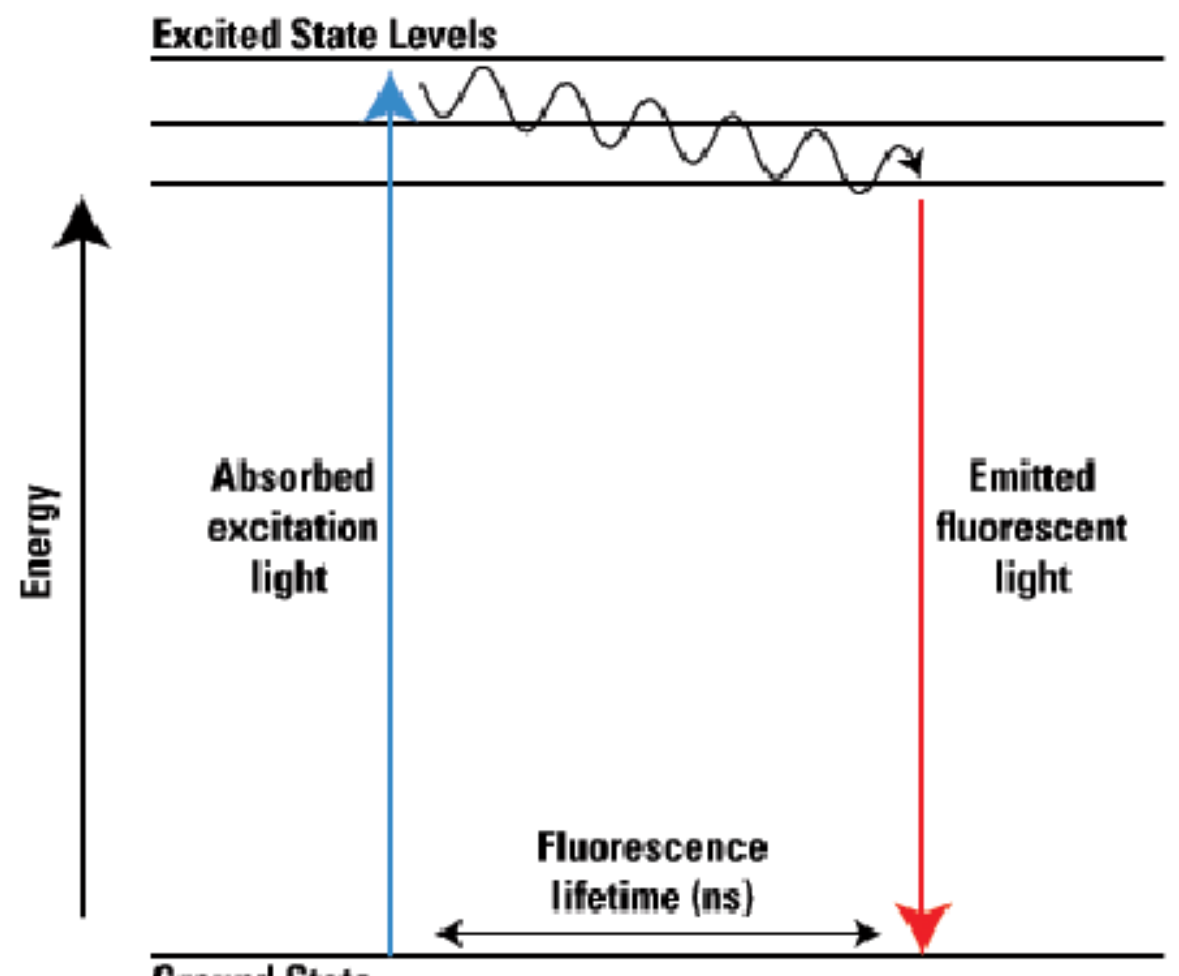

Figure 1: Electron Relaxation and Excitation 
In order to apply QDs to the solar cell, they must be deposited into a material that will act as a carrier. This research utilizes PDMS due to its conformability, availability and low cost [26]. The QDs are synthesized so they fluoresce at or near the band gap of the solar cell and than they are deposited into PDMS which is then applied to the solar cell.

\subsection{Quantum Dots and their Applications}

The research utilizes quantum dots (QDs) made up of CdSe nanocrystals. The CdSe QD core is coated with ZnS which acts as an insulating layer. A quantum dot is a nanometer scale (nanoscale) semiconductor which goes about quantum confinement and confines the electron-hole pair (exciton) in all three directions [3]

Quantum confinement can be observed when the magnitude of a particle's diameter is the same as the magnitude of the electron wave function. The electron wave function described by the Schrodinger equation, depends on space and time and ultimately gives rise to the particle-wave duality.

When the particle's confining dimension is large compared to the wavelength of the particle, the particle can behave as if it were free. The band gap will continue to remain the same as long as the confining dimension remains relatively large but as the particles confining dimensions decrease to the nanoscale, the energy spectrum of the electrons becomes discrete resulting in the band gap becoming dependent on size [4].

This event describes an incident where the electrons and holes are being squeezed in a dimension approaching a critical quantum measurement, called the exciton Bohr radius, which describes the approximate distance between the proton and electron in a hydrogen atom [5]. A quantum dot's excitons are confined in all three dimensions, a 
quantum wire confines excitons in two dimensions and a quantum well confines in one dimension allowing free propagation in two dimensions.

Quantum dots allow one to investigate semiconductors in the finite state regime. As the QD gets smaller the band gap gets larger, meaning more energy is needed to excite electrons across the band gap, resulting in greater photon energy being released as the excited electron goes back to its resting state [6][7]. This can be equated to higher frequency photons being emitted as the QD gets smaller which shifts the color of the light emitted from red to violet, or to a shorter wavelength on the electromagnetic spectrum.

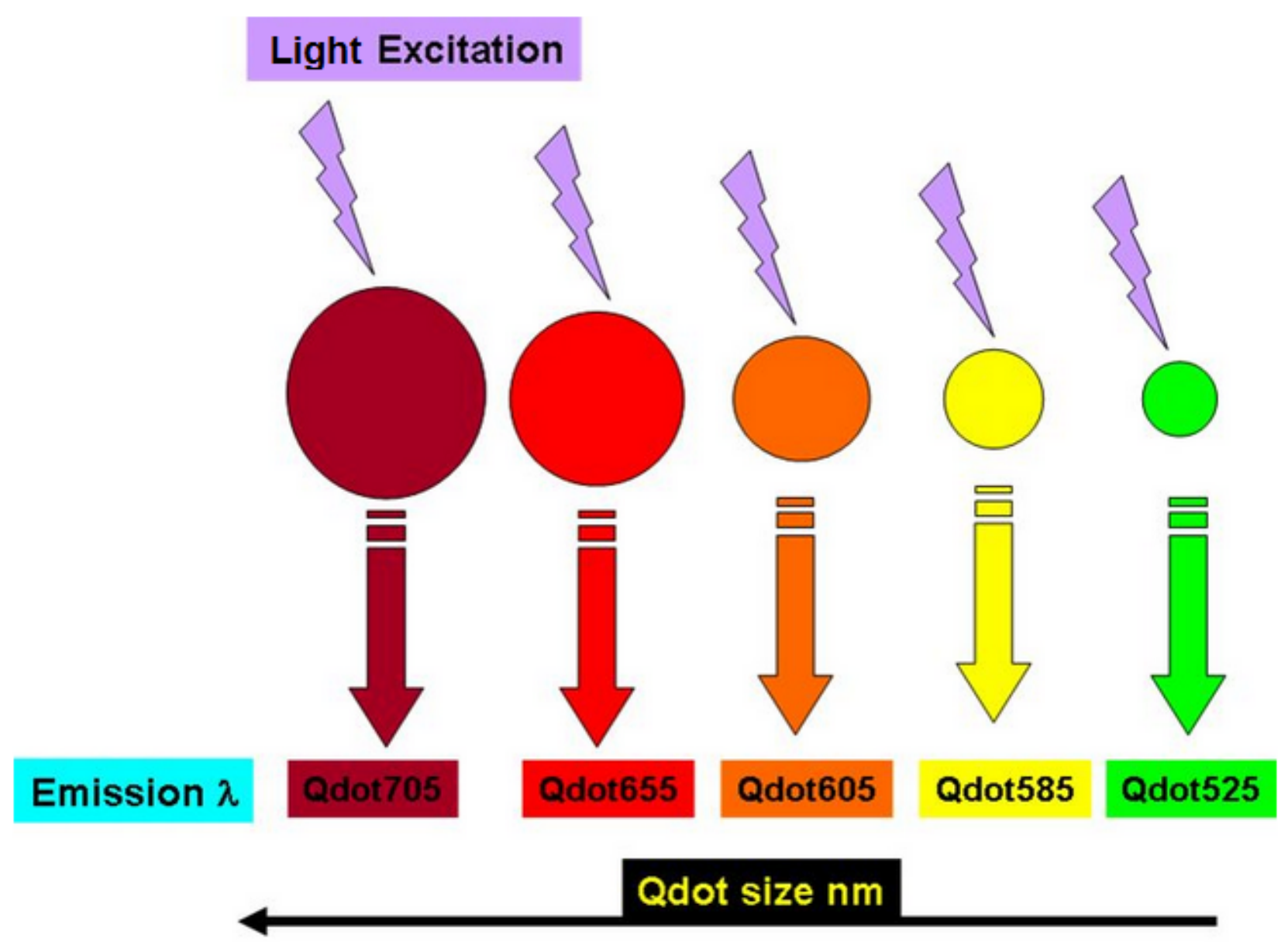

Figure 2: Quantum dot Size and Fluorescing Wavelengths 
QDs can be utilized for different applications because of their ability to reduce the energy of an incoming photon with energy higher than the band gap to the energy of its band gap. A QD that fluoresces in the red region will fluoresce photons with energies in the red region when they are irradiated with any photons of higher energy.

QDs have advantages that are not present in organic dyes. Organic dyes can be used to aid in biological imaging and are typically used to enhance the color of fabric [16]. QDs produce a brighter emission and higher signal to noise ratio [18]. The brightness of QDs can be up to 10-20 times brighter and the fluorescence time of QDs is 10 - 40 nanoseconds which is much greater than the few nanoseconds for an organic dye[18] [19] [20] [21]. The superior properties of QDs make them promising alternatives for fluorescing applications.

QDs can be applied to the biological field as a superior organic tag due to their brightness and stability. Application of QDs in biology include 3D imaging, molecular and cellular tracking in real time [8]. Of particular interest is the use of QDs for in vitro imaging of pre-labeled cells, which can be used for tumor targeting under in vivo conditions.

In computing, quantum dots can be used in solid-state quantum computation. The computation device utilizes quantum mechanical phenomena such as superposition and entanglement to perform operations. Quantum computing uses quantum properties to represent data and perform operations. When applying small voltages to the leads, the flow of electrons through the QD can be controlled which will allow measurements of spin, entanglement, superposition and other properties to be made. With a way of performing operations, quantum calculation can be possible [9]. 
Light Emitting devices can greatly benefit from QDs. Light Emitting Diodes (LEDs) which produce monochromatic light can be applied with QDs and produce light in the desired region. Quantum dots can be used to render the appropriate color in displays, since they can replace the filters currently used to produce red, green and blue pixels allowing the display to be much more efficient since more of the light produced reaches the eye [10]. QDs are also valuable for displays since they emit light in very specific Gaussian spectral distribution which results in a display that produces colors more easily perceived by the human eye.

QDs can be used to increase the efficiency of silicon photovoltaic devices. Since QDs can be synthesized to fluoresce at any region in the visible and infrared spectrum, QDs that fluoresce at a wavelength specific to the band gap of the cell can be applied to allow all photons irradiated upon the cell to be converted to the photons specific to the band gap, thereby increasing the efficiency of the solar cell [4]. Research presented in the Materials Research Society 2008 fall meeting reported that QDs can produce as many as seven excitons from a single solar high energy photon. This production of multiple excitons from a single high energy photon is possible because of the quantization of energy levels in QDs which allows for coulomb coupling, enhanced auger processes and elimination of the requirement to conserve crystal momentum. The production of multiple excitons would allow an increase in the theoretical efficiency from $31 \%$ to $42 \%$ [11]. Further research is being done to test the feasibility of this in large scale silicon photovoltaic production. 


\subsection{Incorporating QDs into Solar Cells}

In order to apply QDs to solar cells the increase in cost associated with the synthesis, deposition and application of the QDs to the solar cells must be less than the cost of attaining the same power by purchasing additional solar cells. The motivation of the application of QDs to solar cells is to increase the power output per unit cell area with a less overall cost then purchasing additional solar cell area or more costly higher efficiency solar cells.

Although there are many applications for QDs, the focus of this research is on the use of QDs to produce photons specific to a particular solar cells band gap. Since QDs can be synthesized to fluoresce at any region in the visible and infrared region of the electromagnetic spectrum, QDs specific to solar cells with band gaps corresponding to wavelengths in those regions can be utilized to increase efficiency. QDs can be applied to all solar cell technologies with band gaps in the visible and infrared region. Solar cells with band gaps in the visible and infrared region include silicon, amorphous silicon, Copper Indium Galium Selenide (CIGS) and Zinc Selenium (ZnSe), Gallium Arsenide cells among many others. 
Table I: Different Solar Cells and their Corresponding Band Gaps

\begin{tabular}{|l|l|l|l|}
\hline Solar Cell Material & Band Gap & Wavelength & Electromagnetic Region \\
\hline Amorphous Silicon & $1.7 \mathrm{eV}$ & $\sim 730 \mathrm{~nm}$ & Red \\
\hline Ga Rich CIGS & $1.6-1.7 \mathrm{eV}$ & $>=730 \mathrm{~nm}$ & Red \\
\hline ZnSe & $2.7 \mathrm{eV}$ & $\sim 460 \mathrm{~nm}$ & Blue \\
\hline Organic & $1-4 \mathrm{eV}$ & $\sim 310-1240 \mathrm{~nm}$ & Various \\
\hline Silicon & $1.1 \mathrm{eV}$ & $\sim 1120 \mathrm{~nm}$ & Infrared \\
\hline Galium Arsenide & $1.43 \mathrm{eV}$ & $\sim 865 \mathrm{~nm}$ & Infrared \\
\hline
\end{tabular}

In order to apply QDs to a solar cell, it is necessary to have a medium or carrier material in which the QDs can be deposited before they are applied to the cell. Various materials can be utilized as carriers for the QDs including Spin on Glass (SOG) and transparent polypropylene but this research will focus on the use of PDMS (Polydimethylsiloxane) as a carrier for the QDs for the reasons mentioned in section 1.1.

\section{Chapter 2 - Synthesis and Application}

\subsection{Quantum Dot Synthesis}

The quantum dots used in this research are ones synthesized using a process developed at California Polytechnic University, San Luis Obispo (Cal Poly) [12]. This method is different from other synthesis methods because it utilizes a silicone oil bath for cooling. To make the CdSe core, the precursors selenium trioctylphosphine (Se-TOP) and cadmium (Cd) were synthesized using the methods in the procedure. The standard 
procedure calls for selenium powder and cadmium oxide powder; once these materials are reacted and are at the desired target temperature they are then synthesized at different time intervals depending on the core size desired. The standard procedure indicates a synthesis temperature of $160{ }^{\circ} \mathrm{C}$, however it does not indicate at what time the QDs should be removed. Trials with different removal times of $30 \mathrm{~s}, 60 \mathrm{~s}$ and 90 s while keeping all other parameters constant were performed resulting in different core sizes. Table II indicates the fluorescing wavelength at different removal times [28].

Table II: Removal Times and Quantum Dot Size.

\begin{tabular}{|l|l|l|l|}
\hline Time (s) & 30 & 60 & 90 \\
\hline Fluorescing & 518 & 539 & 550 \\
Wavelength $(\mathrm{nm})$ & & & \\
\hline
\end{tabular}

The synthesis of the $\mathrm{ZnS}$ shell requires a reaction of $\mathrm{ZnS}$ with the previously synthesized CdSe. The CdSe core and the ZnS were allowed to react at a temperature between $110{ }^{\circ} \mathrm{C}$ and $160{ }^{\circ} \mathrm{C}$. Once the reaction had proceeded and the solution had cooled, the resulting product is irradiated with an ultraviolet (UV) light to quantify the concentration of QDs in the solution and to determine the wavelengths at which they fluoresce.

A spectrophotometer is used to quantify the QDs in solution. UV light is irradiated on the QDs in solution at a 90 degree angle and the intensity of the fluorescence produced by them is detected by the spectrophotometer and reported. It has been found that there is a linear relationship between the concentration of QDs in solution and the intensity of fluorescence as detected by the spectrophotometer. This method also allows the user to 
determine the peak wavelength at which the QDs fluoresce. The set up of the spectrometer is depicted in Figure 3.

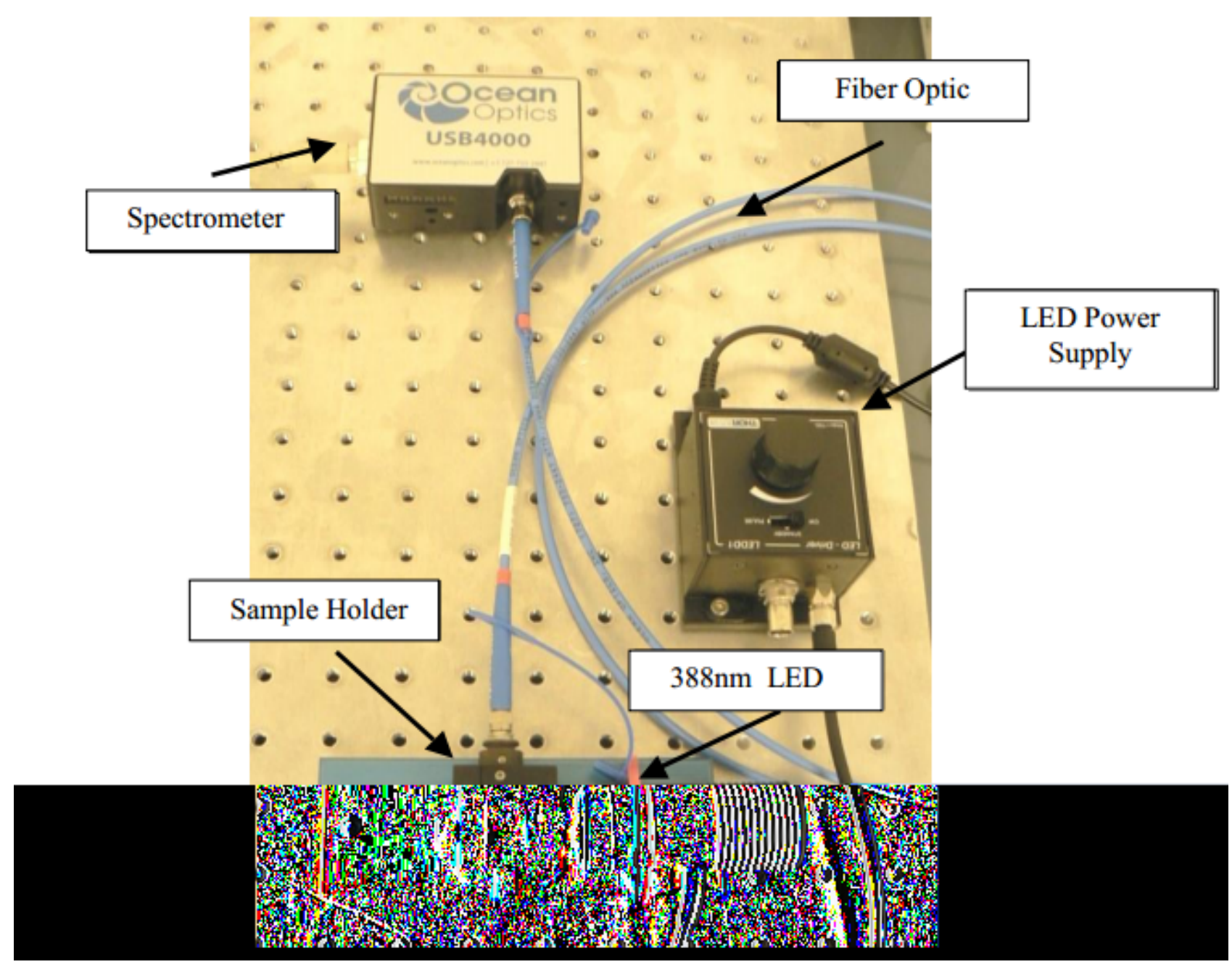

Figure 3: Spectrometer Set Up [28]

\subsection{PDMS as a Medium to Carry QDs}

PDMS (Polydimethylsiloxane) is a polymeric organosilicon and is the most widely used silicon polymer [14]. PDMS has a band gap of $\sim 4.77 \mathrm{eV}$ so it is transparent to light in the visible and UV with wavelengths longer than $260 \mathrm{~nm}$ [29]. PDMS is also chemically inert and weather resistant. Applications include its use in contact lenses, medical devices and as an elastomer [30]. PDMS has some important mechanical properties; it is 
viscoelastic so given a long flow time, or high temperature it will mold to the imperfections of a surface. This mechanical property allows PDMS to be easily applied to many surfaces as a protective layer or as a carrier of certain substances. PDMS is commonly used in soft lithography so it's one of the common materials used for flow delivery in microfluidic chips [31]. One of the characteristics of PDMS that makes it especially attractive is its low cost; PDMS can be purchased at $\$ 2-4.5 / \mathrm{kg}$ depending on the vendor and quantity purchased.

Due to the properties of PDMS, its availability and low cost, it is an attractive material to be used as a carrier. This work utilizes PDMS as a medium to carry the QDs; therefore data collected from solar arrays applied with QDs include any attenuation or efficiency loss associated with PDMS when subjected to solar irradiation.

\subsection{Necessary QD State for PDMS Deposition}

The QD synthesis process developed at Cal Poly utilizes octadecene as a solution for the Se-TOP when making the CdSe precursor, for this reason the final QDs are in an octadecene solution. PDMS is typically cured by combing a silicone elastomer base and a silicone elastomer curing agent at a 10:1 ratio. Once both precursors of PDMS are combined and the mixture is subjected to a temperature at or near $75{ }^{\circ} \mathrm{C}$ for 45 minutes, a strong PDMS backbone with siloxane linkages is formed. Cross-linked PDMS is made up of lower molecular weight and uncross-linked oligomers that make up the bulk polymer. Certain reactive polymers will react with PDMS and break it down into smaller subunits. Solvents such as acids if allowed to react with PDMS will completely dissolve the PDMS after 24 hours and leave a white precipitate. The products of this reaction are made up of oligomers with different numbers of dimethylsiloxane subunits. Organic solvents, although 
not as reactive will also dissolve PDMS by breaking the $\mathrm{Si}$ and $\mathrm{O}$ bonds. These chemicals will dissolve fully cured PDMS and will prevent uncured PDMS from curing which poses a problem when attempting to suspend additional compounds into PDMS, since the QDs are dissolved in octadecene after sythesis [15].

When PDMS is in contact with an organic solvent, the solvent may extract the uncross-linked oligomers from the bulk [15]. This causes a problem since the QDs once synthesized are in octadecene which if added to PDMS during the curing process will cause the PDMS to fail to cure or only partially cure. An additional issue is the degree of solubility of octadecene, greater volumes of solvent need to be added in order to solubilize the QDs. In order to mitigate the issue associated with adding organic solvents to PDMS, a different solvent must be used which has greater solubility then octadecene to minimize the volume added. Toluene has greater solubility which will decrease the amount of solvent used and will facilitate its addition into the PDMS compound. In order to get the QDs into toluene they must be extracted from the PDMS, dried and then dissolved into toluene with minimal solvent used. The process used to extract the QDs from octadecene to allow them to dry and subsequently dissolve them into toluene is described in later sections.

\section{Chapter 3 - Experiments and Results}

\subsection{QD Extraction from Octadecene}

As previously described the QDs must be removed from octadecene in order to be dried and dissolved into another solution. In order to extract the QDs from octadecene a solvent that will react with the octadecene only and not the QDs is necessary. For this 
reason ethanol is added to the QD + octadecene solution and then centrifuged. Due to the much higher polarity of alcohols as compared to alkenes, this process leads to the emulsion of both solvents. The QDs remain at the bottom of the centrifuge tube while the emulsified ethanol + octadecene mixture remain at the top of centrifuge tube. A pipette can then be used to extract the emulsified mixture while the QDs remain. This process may have to be repeated with further addition of ethanol and centrifuging until the QDs appear to coagulate on the inner surface of the centrifuge tube.

The QDs on the inner surface of the centrifuge tubes will still have remnants of the octadecene and ethanol solvents on them so the centrifuge is heated to $\sim 80{ }^{\circ} \mathrm{C}$ while being applied with a flow of dry air to allow for evaporation of the solvents and complete drying of the QDs. Once the QDs have completed drying they can then be dissolved in toluene and subsequently added to PDMS. QDs are capable of dissolving in toluene up to $30 \mathrm{mg} / \mathrm{ml}$ making it possible to dissolve much larger amounts of QDs in toluene and producing a much greater concentration in toluene as compared to octadecene. The greater concentration of QDs in toluene allow for greater amounts of QDs to be deposited into the PDMS with minimal toluene volume.

\subsection{QD Absorption with Time}

Before attempting to apply QDs to solar cells it is important to verify that the QDs will continue to fluoresce with the same intensity over time. Since solar cells are intended for use over long periods of time it is absolutely necessary that the QDs maintain their fluorescing efficiency and output over the life of the solar cell. In order to test the stability of QD over time, QDs of different concentrations were deposited into cured and uncured 
PDMS. The uncured PDMS was just made up of the silicone elastomer base without the added silicone elastomer curing agent. The QD intensity was measured over a 3 month period using a spectrophotometer with re-measuring occurring every two days. The figure below shows the intensity of QDs of different concentrations over a 3 month period.

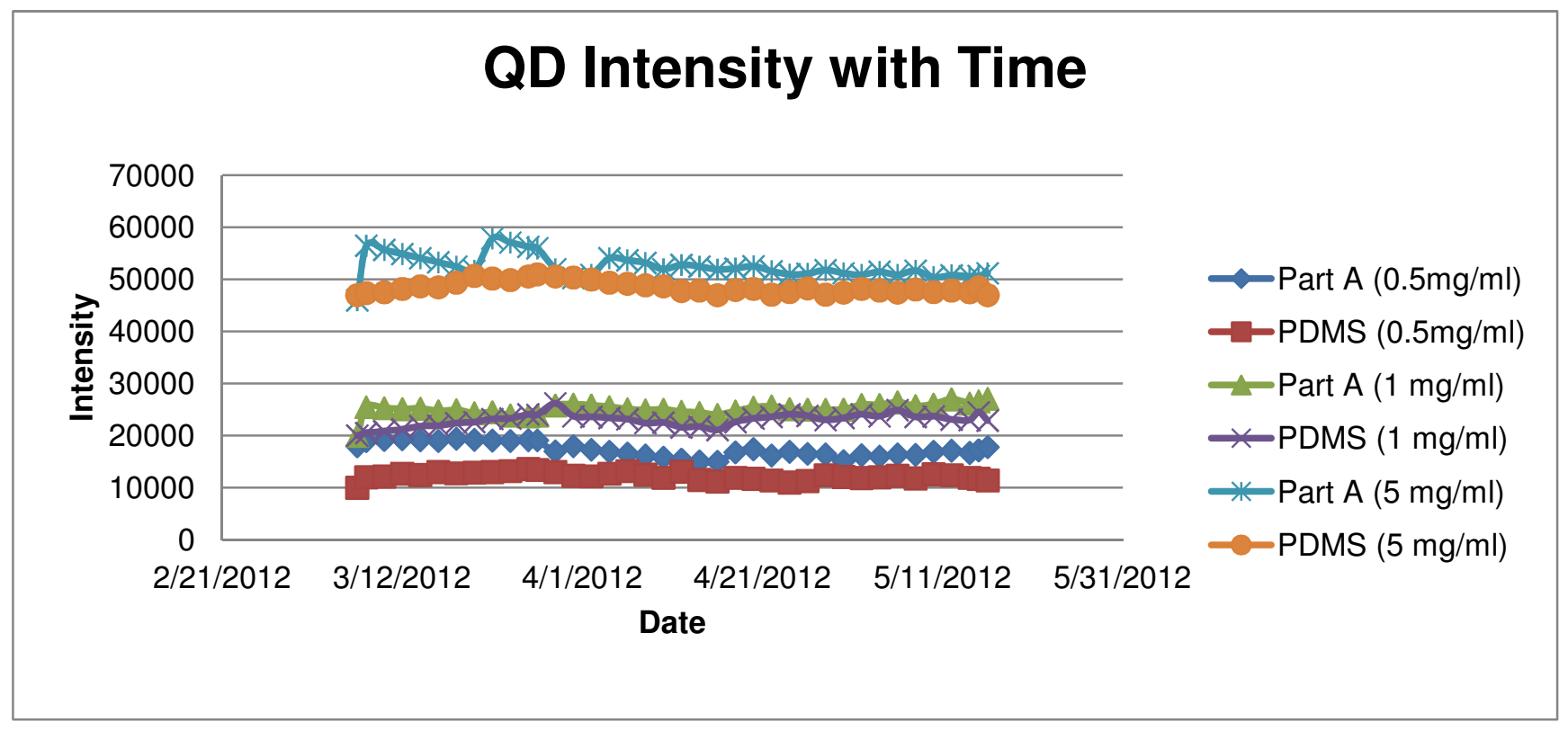

Figure 4: QD intensity over a 3 month Period

In order to ensure that the QDs will maintain their intensity in various mediums, QDs shelled with ZnS were deposited in cured and uncured PDMS and measurements of QDs of different concentrations and medium were measured six times for each individual concentration on the same day with the same spectrophotometer. The PDMS and PDMS base were placed in a cuvette with dimensions of $12.5 \mathrm{~mm}$ by $12.5 \mathrm{~mm}$ by $45 \mathrm{~mm}$, once the walls are excluded this allows for a little over $10 \mathrm{~mm}$ of thickness of PDMS or PDMS base. The data in Figure 3 indicate that the QDs on average lost $0.4 \%$ of intensity while being deposited in a substrate such as PDMS over a 3 month period. The standard deviation of the data is $0.99 \%$ of the average, indicating that the data will fluctuate by at least $0.99 \%$ above or below the average, therefore the data is validated over a large sample size. The 
loss of intensity of QDs over time can be partially attributed to the fluctuation in the data reported. From the above data, it can be concluded that the QDs remain relatively stable over time, however additional experiments would need to be performed to ensure that the QDs will remain stable for extended periods of time such as years. Although this trial does not prove the long-term stability of QDs, it indicates that there is minimal degradation over the course of a few months, which is essential due to the lifetime of the applications that QDs will potentially be paired with.

The stability and quantum yield of QDs can be attributed to the ZnS shell. Unshelled QDs are much more susceptible to degradation and decreased quantum yield. Experiments done at the German Institute of Materials Science and Research and Testing indicate that quantum yield increases from less than $5 \%$ for unshelled QDs to greater than $50 \%$ with an increased $\mathrm{ZnS}$ coating thickness of $0.6-0.8 \mathrm{~nm}$ [22].

Additionally, Figure 3 indicates that QDs of greater concentration have greater intensity; this method can be used to determine varying concentrations of QDs. Further experiments to determine the extent of this correlation have been done and will be explained in later sections.

\subsection{QD Intensity with Concentration}

When using QDs for various applications it is important for one to determine the number or volume of the QDs being used. Due to the nanoscale size of the QDs it is impossible to determine the number of QDs present in a volume, for this reasons it is easier to work with volumes of QDs. Since the mass of QDs used is typically in the milligrams range, it is difficult to measure QDs by volume so they are much more easily 
weighed. If one wishes to determine the volume of the QDs being used, this can be determined from the known density of the QDs. The spectrophotometer measures intensity of the QDs when they are subjected to UV light directed normal to them. The energy of the photons emitted from the QDs can be detected by the spectrophotometer. It is then a logical conclusion that with a greater QD concentration the intensity of photons emitted will increase. The rate at which intensity increases with increasing QD concentration was unknown, therefore trials needed to be conducted to determine the correlation. Since the synthesis and extraction of QDs require much more resources and time, initial experiments were done with fluorescent dye (fluorescein). The figure below reveals the correlation between an increasing concentration of fluorescein in toluene and the corresponding fluorescent intensity.

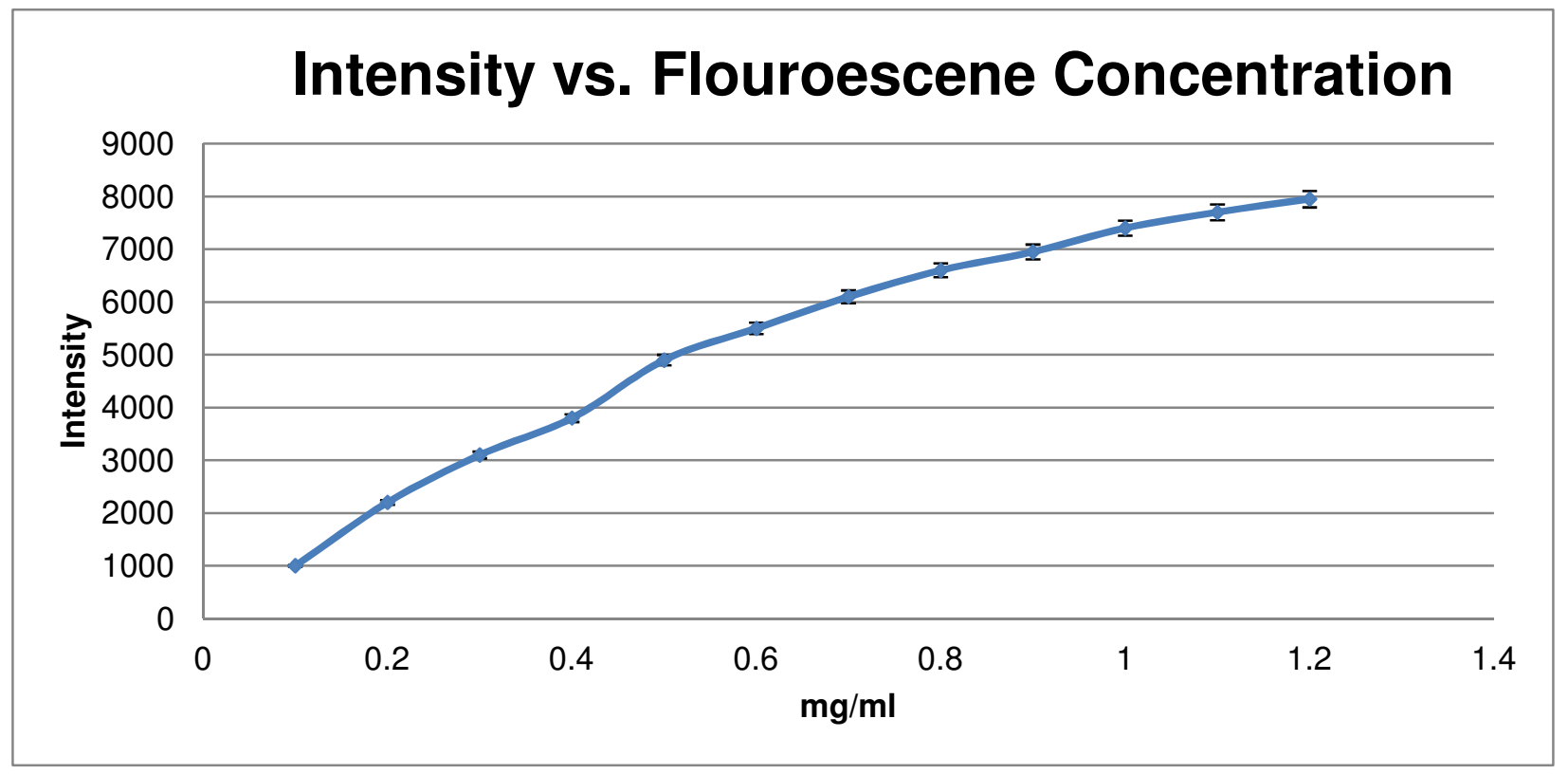

Figure 5: Intensity of Fluorescein when Subjected to UV Light at Different Concentrations

Flourescein was weighed out in increments of $0.1 \mathrm{mg}$ up to $1.2 \mathrm{mg}$ and the intensity was measured six times for each concentration using a light spectrometer with the set up 
shown in Figure 3. The error bars shown on the plot indicate twice the standard deviation. The relationship between intensity and concentration is not continuous, when the concentration of fluorescein exceeds $\sim 0.7 \mathrm{mg} / \mathrm{ml}$, the intensity does not continue to increase at the same rate. With further increase in concentration, intensity begins to decrease. The decrease in intensity is due to interference caused by the high concentration of fluorescein atoms that block the incoming UV light from their neighbors in addition to the fluorescing photons being self-absorbed. Maximum photon emission can occur if every fluorescein atom is irradiated with light and is allowed to excite an electron from the valence to the conduction band. With increasing fluorescein concentration some of the fluorescein atoms are prevented from excitation because their neighbors block the incoming light. Due to the intensity loss that occurs at higher concentration, a relationship between intensity and concentration is determined at concentrations below $0.7 \mathrm{mg} / \mathrm{ml}$, this data is plotted in Figure 6.

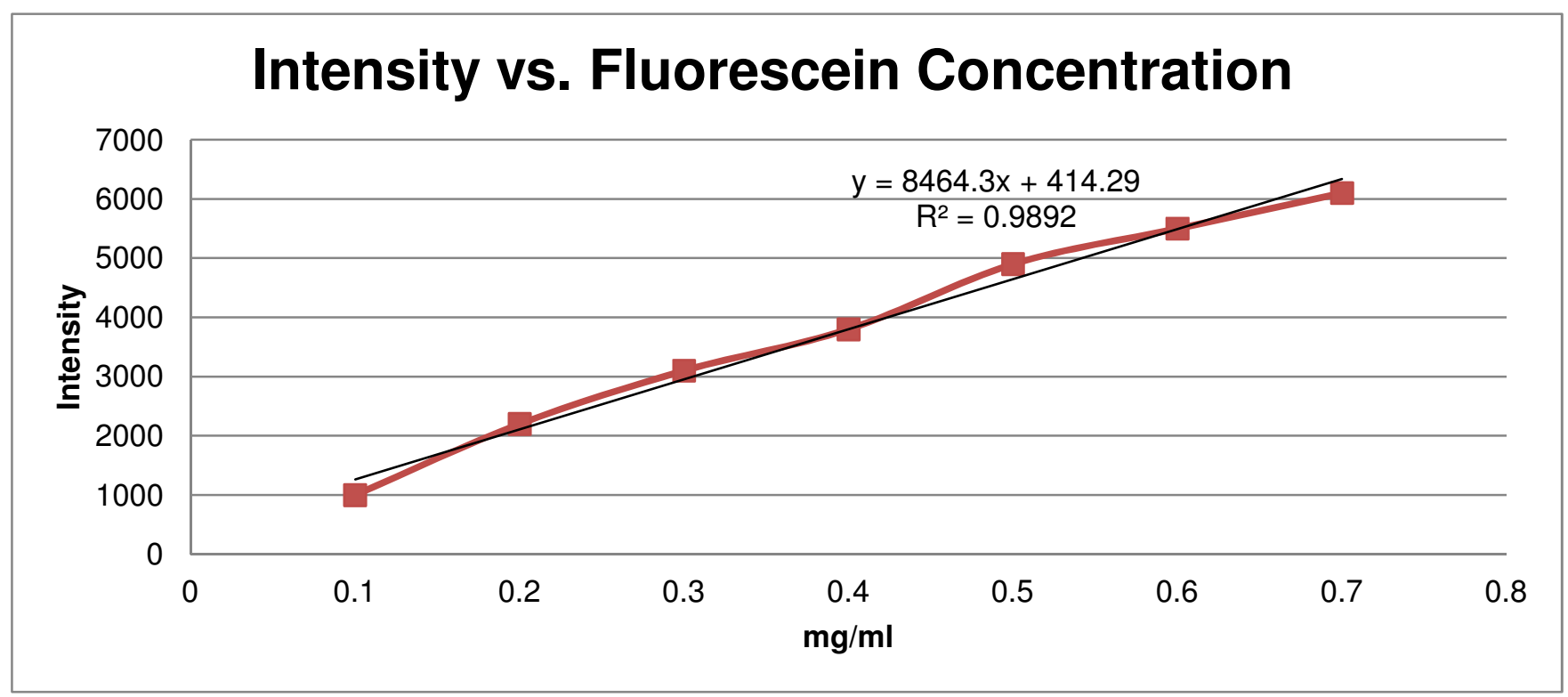


Figure 6: Intensity of Fluorescein when Subjected to UV light at different Concentrations in the Linear Region

This data indicates that the there is a linear correlation between the intensity measured by the spectrophotometer and the concentration of the fluorescein. The above data is the result of the mean of six samples measured during the same day. Using this relationship, the concentration of synthesized QDs in solution can be determined by simply measuring the intensity, making extraction of QDs for this purpose unnecessary.

A similar trial is done with QDs extracted and re-dissolved in toluene at the desired concentrations to determine the correlation between an increase in QD concentration and intensity upon irradiation by a UV light source. The figure below indicates that the QDs of increasing concentration behave similarly to the fluorescein. Figure 7 reveals the interference and self-absorption phenomena described earlier as the QD concentration continues to increase, there is a decrease in the rate at which the intensity increases. 


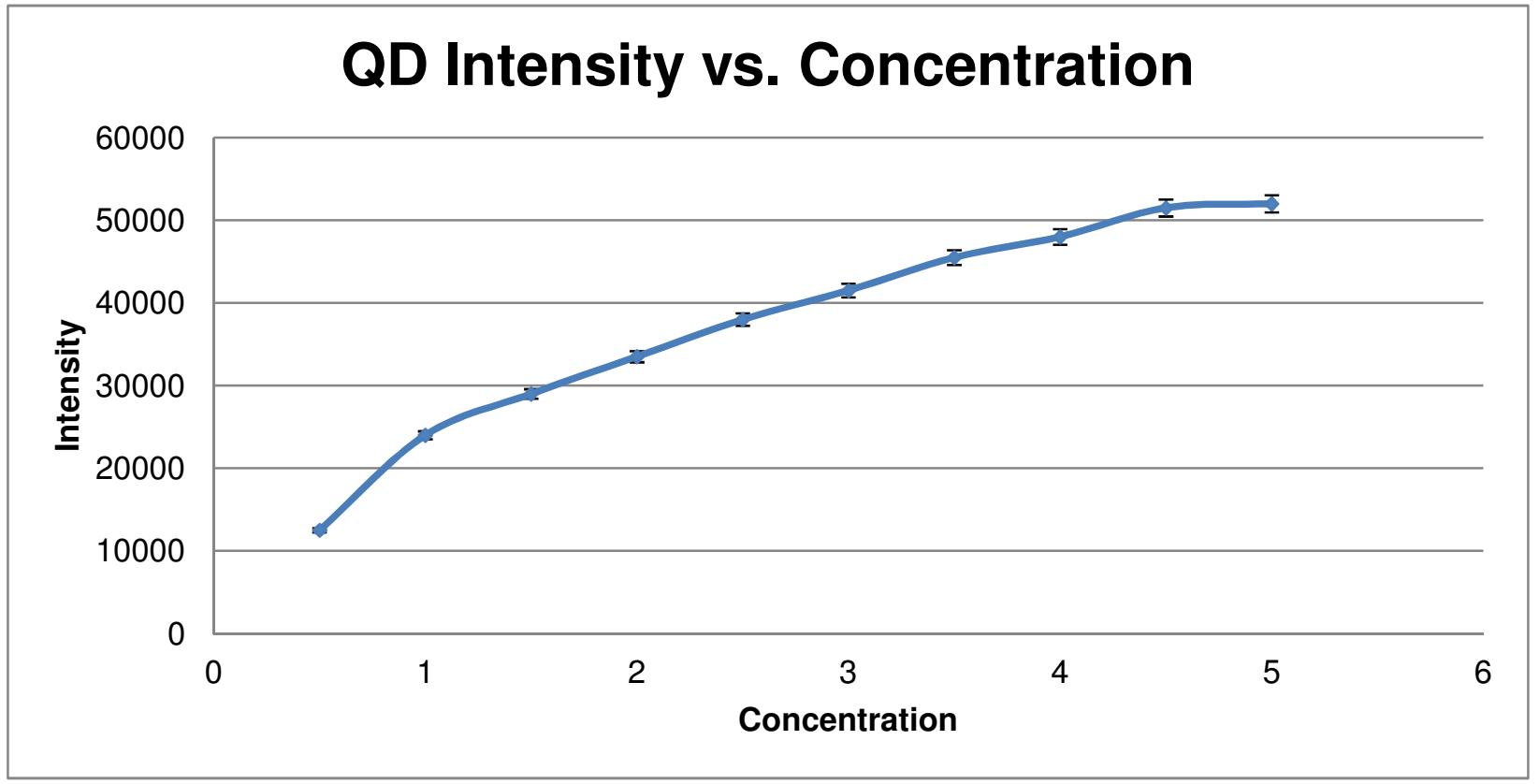

Figure 7: QD intensity with concentration increasing in $0.5(\mathrm{mg} / \mathrm{ml})$ intervals up to 5 $(\mathrm{mg} / \mathrm{ml})$

All data points in Figure 7 are an average of 6 data points. The error bars on the graph are made up of twice the standard deviation of the data points. Figure 7 raises a question on the linearity of the Intensity vs. Concentration function. Is this function at any point linear? It appears to be a lot more linear between concentrations of $0.5 \mathrm{mg} / \mathrm{ml}$ and 1 $\mathrm{mg} / \mathrm{ml}$. For this reason the intensity is measured in this region at increasing concentrations at $0.1 \mathrm{mg} / \mathrm{ml}$ increments. Figure 8 indicates that there is a much greater degree of linearity in this region. 


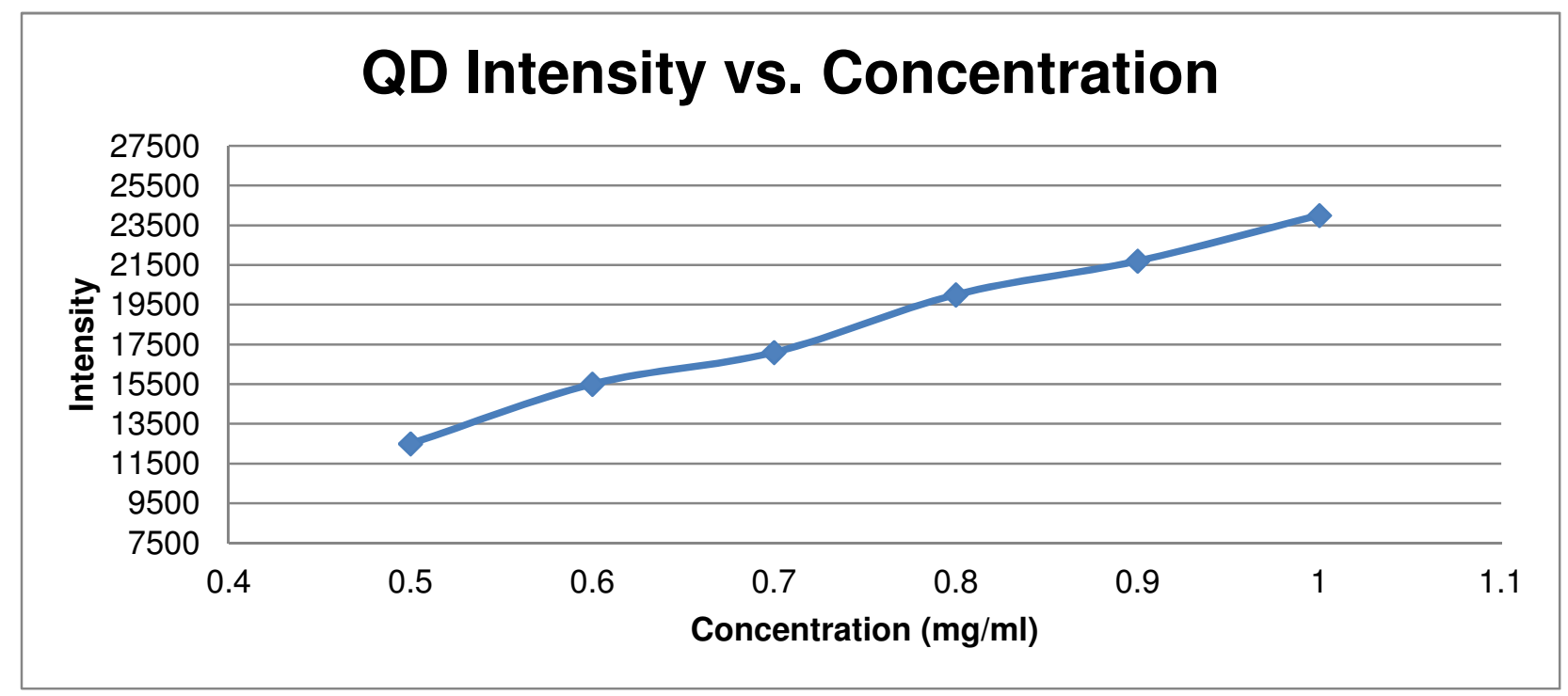

Figure 8: QD intensity with concentration increasing in $0.1(\mathrm{mg} / \mathrm{ml})$ intervals up to 1 $(\mathrm{mg} / \mathrm{ml})$ in the Linear Region

Figure 8 indicates that there is a linear relationship between the intensity and the concentration of the QDs. As in the case of the fluorescein, in the linear region, the intensity of the QD can be used to determine the concentration making it unnecessary to extract and dry the QDs to determine the concentration of the initial QD solution. From figures 7 and 8 it can be deduced that the intensity at which the QDs fluoresce continues to increase beyond the linear region but as the concentration continues to increase beyond this region, the greater the interference and the less efficient the QDs are at fluorescing.

\subsection{Intensity of QDs + PDMS}

Since PDMS is the medium in which the QDs are to be deposited, it is necessary to measure the intensity of the QDs while they are deposited into PDMS in order to quantify the degree of attenuation, if any, which is caused by the PDMS. QDs made from the batch were extracted and deposited into the PDMS while others were extracted and deposited 
into toluene. The intensity of the QDs in either toluene or PDMS is measured and compared in order to quantify any attenuation caused by the PDMS. Figure 9 indicates that there is some degree of attenuation caused by the PDMS.

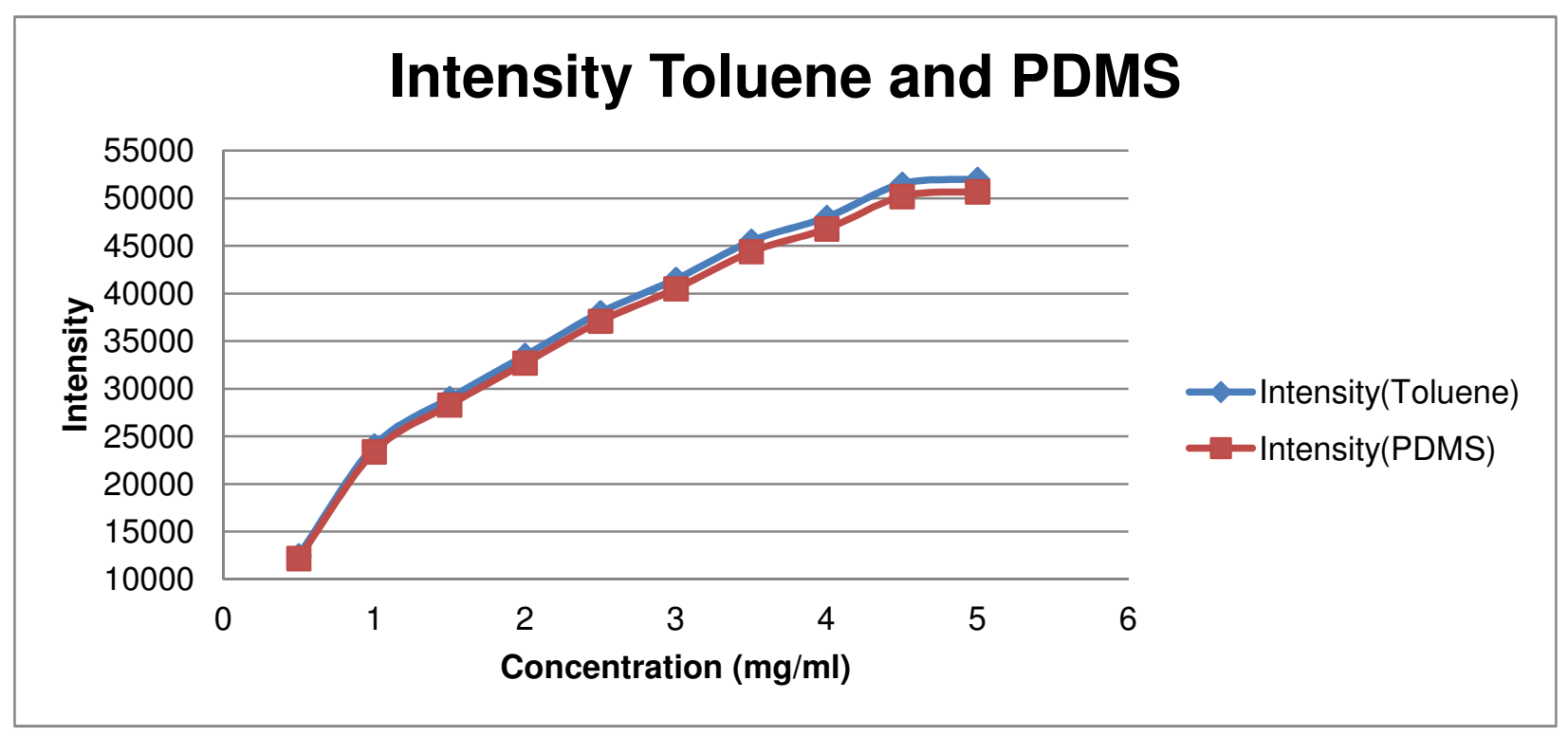

Figure 9: A comparison of QD intensity in either toluene and PDMS

As seen in Figure 7, there is a decrease in the rate at which intensity is increased with an increase in concentration. The intensity of the QDs in PDMS appears to be consistently less than its intensity in other solvents such as toluene. Data in Table II below indicate that there is a $\sim 2.7 \%$ decrease in intensity of the QDs of the same concentration when they are deposited in $5 \mathrm{ml}$ thick layer PDMS instead of another solution. 
Table III: A Comparison of QDs when deposited in either PDMS or Toluene

\begin{tabular}{|c|c|c|}
\hline Intensity(Toluene) & Intensity (PDMS) & I(PDMS)/I(Toluene) \\
\hline 12500 & 12100 & $96.80 \%$ \\
\hline 24000 & 23400 & $97.50 \%$ \\
\hline 29000 & 28300 & $95.59 \%$ \\
\hline 33500 & 32200 & $96.12 \%$ \\
\hline 38000 & 37100 & $97.63 \%$ \\
\hline 41500 & 40500 & $97.59 \%$ \\
\hline 45500 & 44400 & $97.58 \%$ \\
\hline 48000 & 46800 & $97.50 \%$ \\
\hline 51500 & 50200 & $97.48 \%$ \\
\hline 52000 & 50700 & $95.50 \%$ \\
\hline Mean & & $97.33 \%$ \\
\hline Stdev & & $0.87 \%$ \\
\hline
\end{tabular}


The attenuation of intensity of the QDs when deposited in PDMS proposes an issue when using PDMS as a medium for the QDs on the solar cells since there is a consistent attenuation caused by the PDMS. Further trials are done on a solar cell in order to quantify the power loss caused by attenuation of the QDs in PDMS.

In order to facilitate the quantification of the attenuation loss, a similar trial was conducted in the linear region. The difference in intensity of the QDs when deposited in Toluene or PDMS is not dependent on the concentration of QDs in solution.

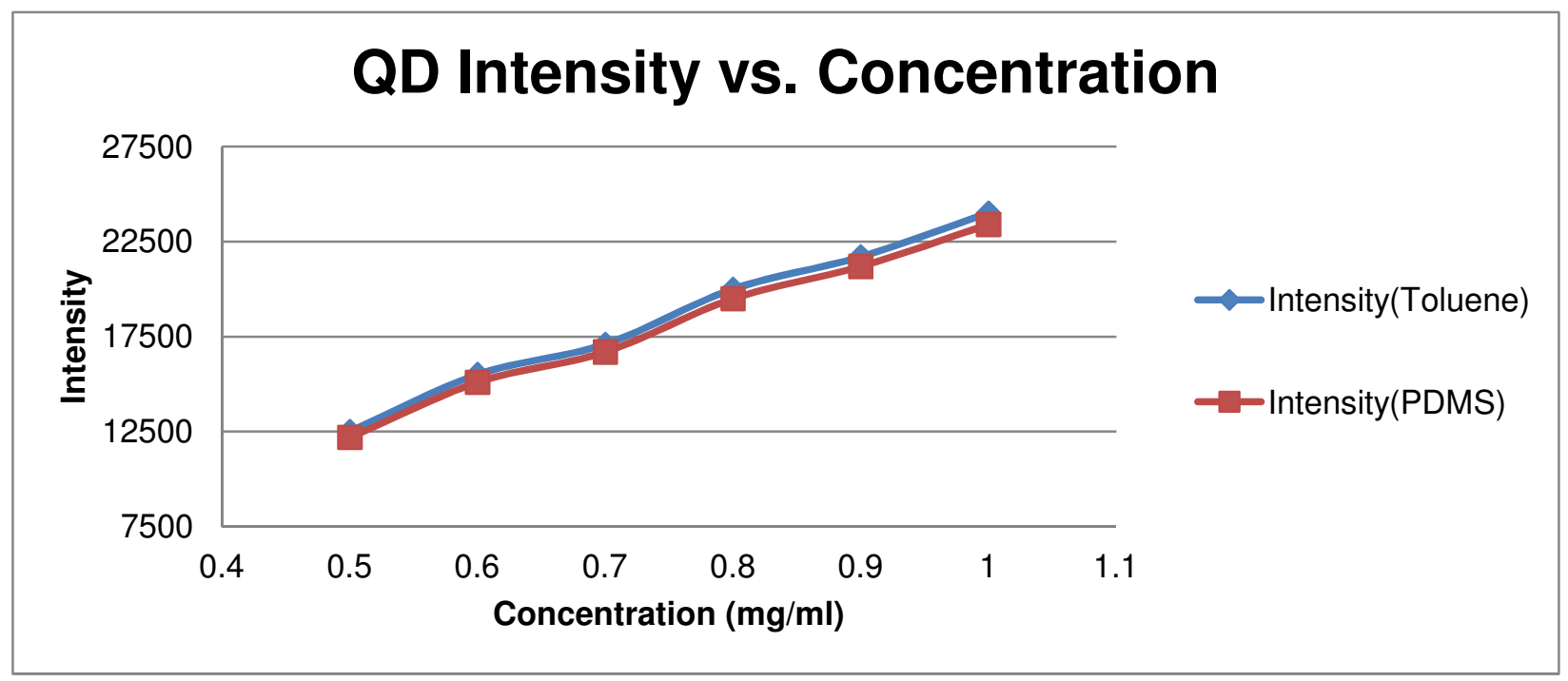

Figure 10: A comparison of the intensity QDs in either toluene or PDMS in the linear region

As in Figure 9, Figure 10 indicates that there is a consistent attenuation of the QDs when they are deposited into PDMS, this will be explained further in section 4.4.

\subsection{Solar Cell Efficiency when Applied with QDs + PDMS}

Trials with QDs applied to a solar cell were done using an amorphous silicon solar cell which has a band gap of $1.7 \mathrm{eV}$. Using this band gap and the energy wavelength 
relationship described by Equation 1 this band gap can be translated into a wavelength.

$$
E=h c / \lambda
$$

- $E$ is energy in eVs

- $\mathrm{h}$ is Planck's constant and is equal to $6.62606896(33) \times 10-34 \mathrm{~J} \mathrm{~s}$ or

$$
4.13566733(10) \times 10-15 \mathrm{eV} \mathrm{s}
$$

- $\mathrm{C}$ is the speed of light and is equal to $299,792,458 \mathrm{~m} / \mathrm{s}$

- $\lambda$ is the wavelength and is expressed in $\mathrm{m}$

Using equation 1 the band gap of $1.7 \mathrm{eV}$ is equivalent to $\sim 729 \mathrm{~nm}$ which is at a slightly lower energy then the red region. For this reason as described in section 1.2, the efficiency of the solar cell is optimized when it is applied with QDs that fluoresce in the red region so the photons that are being irradiated upon the solar cell are of the same energy as the band gap. The following trials were done with QDs that fluoresce in the orange region due to the lack of availability of large amounts of QDs fluorescing in the red region. This will diminish the effect since the photons are fluorescing in a region that produces photons farther away from the band gap of the solar cell.

Since it is necessary to determine if the incoming light is being attenuated by the PDMS before it reaches the solar cell, it is necessary to first compare the power produced by a solar cell with just PDMS as compared to one without anything applied to it to determine the source of the loss if any. Figure 9 reveals the maximum power produced by solar cells 
when applied with PDMS or PDMS + QDs and this is compared to solar cells without any application.

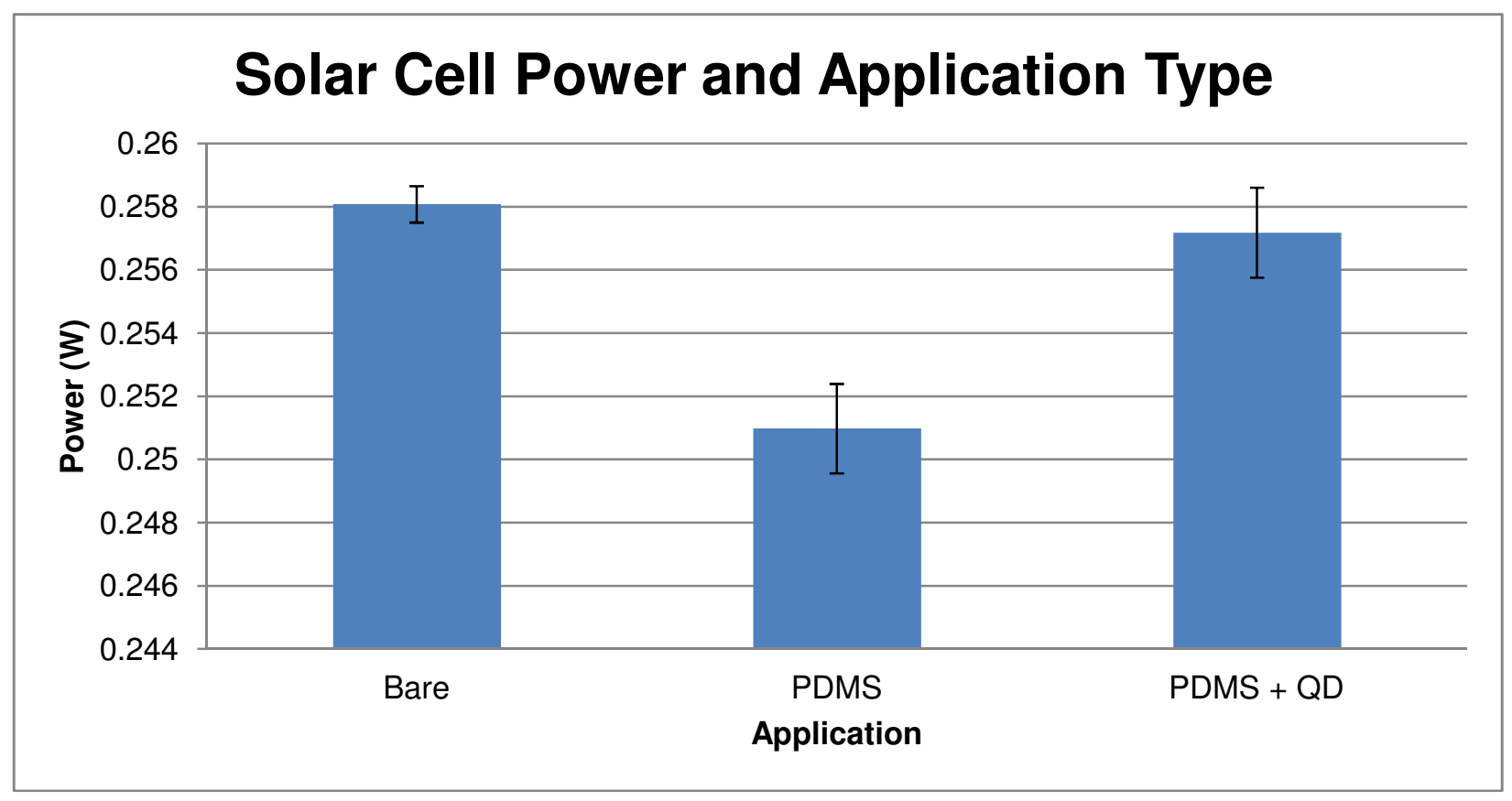

Figure 11: Solar cell power bare and compared with an application of PDMS or PDMS +

QDs

The data collected in Figure 11 was done using a commercially available solar cell with maximum power of $250 \mathrm{~mW}$ and a fill factor of $71.025 \%$. Data was collected using Keithley 2400 Electrometer with Labview software. Voltage and current characteristics were recorded in steps of $10 \mathrm{mV}$ with a wait time of $250 \mathrm{~ms}$. The solar cell was illuminated with an artificial solar spectrum using a Tungsten Halogen lamp. The QDs in the PDMS were deposited at a concentration of $4 \mathrm{mg} / \mathrm{ml}$.

Figure 11 indicates that the application of PDMS on a solar cell causes a decrease in the maximum power produced by the solar cell due to the attenuation of light irradiated upon the solar cell. The panel applied with QD + PDMS reveals that the addition of QDs to 
PDMS results in an increase in power. Since the attenuation caused by the application of the PDMS is greater than the power gained by adding QDs, the application of the QDs deposited in PDMS onto the solar cell results in an overall slight decrease in the maximum power produced by the solar cell.

Table IV: Solar Cell Max Power Bare and Applied with PDMS

\begin{tabular}{|c|c|c|}
\hline Pmax [W] (Bare) & Pmax [W] (PDMS) & Pmax [W] (PDMS)/Pmax(Bare) \\
\hline 0.25709856 & 0.2505902 & $97.47 \%$ \\
\hline 0.25739856 & 0.250516777 & $97.33 \%$ \\
\hline 0.25855224 & 0.251820844 & $97.40 \%$ \\
\hline 0.25811448 & 0.253088129 & $98.05 \%$ \\
\hline 0.258523639 & 0.253545089 & $98.07 \%$ \\
\hline 0.257482434 & 0.250645178 & $97.34 \%$ \\
\hline 0.258627336 & 0.249015786 & $96.28 \%$ \\
\hline 0.258627699 & 0.24994164 & $96.64 \%$ \\
\hline 0.257885969 & 0.250190003 & $97.02 \%$ \\
\hline 0.258459025 & 0.250412166 & $96.89 \%$ \\
\hline Mean & & $97.25 \%$ \\
\hline Stdev & & $0.57 \%$ \\
\hline
\end{tabular}

The results in Table IV denote that there is a $\sim 2.75 \%$ decrease in the maximum power produced by the solar cell when it is applied with just PDMS. Due to the known decrease in power caused by the application of the PDMS, any increase in power after the deposition of the QDs into the PDMS can be attributed to the QDs. The data in Table IV 
indicates that there is an increase in the maximum power produced once the panel is applied with QDs deposited into PDMS.

Table V: Solar Cell Max Power Applied with PDMS and PDMS + QDs

\begin{tabular}{|c|c|c|}
\hline Pmax [W] (PDMS) & $\begin{array}{l}\text { Pmax }[W](Q D+ \\
\text { PDMS) }\end{array}$ & $\begin{array}{l}\text { Pmax [W] (QD + } \\
\text { PDMS)/Pmax(PDMS) }\end{array}$ \\
\hline 0.2505902 & 0.255749088 & $102.06 \%$ \\
\hline 0.250516777 & 0.254220456 & $101.48 \%$ \\
\hline 0.251820844 & 0.25654584 & $101.88 \%$ \\
\hline 0.253088129 & 0.25684175 & $101.48 \%$ \\
\hline 0.253545089 & 0.257039524 & $101.38 \%$ \\
\hline 0.250645178 & 0.257747574 & $102.83 \%$ \\
\hline 0.249015786 & 0.25786392 & $103.55 \%$ \\
\hline 0.24994164 & 0.25849968 & $103.42 \%$ \\
\hline 0.250190003 & 0.25873524 & $103.42 \%$ \\
\hline 0.250412166 & 0.25855932 & $103.25 \%$ \\
\hline Mean & & $102.48 \%$ \\
\hline Stdev & & $0.86 \%$ \\
\hline
\end{tabular}

After the deposition of QDs into the PDMS, there is a $~ 2.5 \%$ increase in the median maximum power produced by the solar cell. A sign test indicates that the median of the data in the (QD + PDMS) series is greater than the median of the data in the (PDMS) series with a p-value of 0.0009 . Since PDMS causes attenuation, this experiment would 
need to be repeated using a medium other than PDMS to confirm that there is a possible $2.5 \%$ increase in power on a solar cell. This will be further discussed in later sections.

Table $\mathrm{V}$ indicates that there is a slight decrease in the power of the panel when it is applied with QD + PDMS compared to the power produced by a bare panel.

Table Vl: Solar Cell Max Power Bare and Applied with PDMS + QDs

\begin{tabular}{|c|c|c|}
\hline Pmax [W] (Bare) & $\begin{array}{l}P \max [W](Q D+ \\
\text { PDMS) }\end{array}$ & $\begin{array}{l}\text { Pmax [W] (QD } \\
+ \text { PDMS)/Pmax(Bare) }\end{array}$ \\
\hline 0.25709856 & 0.255749088 & $99.48 \%$ \\
\hline 0.25739856 & 0.254220456 & $98.77 \%$ \\
\hline 0.25855224 & 0.25654584 & $99.22 \%$ \\
\hline 0.25811448 & 0.25684175 & $99.51 \%$ \\
\hline 0.258523639 & 0.257039524 & $99.43 \%$ \\
\hline 0.257482434 & 0.257747574 & $100.10 \%$ \\
\hline 0.258627336 & 0.25786392 & $99.70 \%$ \\
\hline 0.258627699 & 0.25849968 & $99.95 \%$ \\
\hline 0.257885969 & 0.25873524 & $100.33 \%$ \\
\hline 0.258459025 & 0.25855932 & $100.04 \%$ \\
\hline Mean & & $99.65 \%$ \\
\hline Stdev & & $0.44 \%$ \\
\hline
\end{tabular}


Due to the power loss caused by the application of the PDMS and the power gain caused by the deposition of QDs into the PDMS, there is an overall loss of $\sim 0.35 \%$. This result indicates that there is an expected gain of power if the attenuation caused by the PDMS was removed.

In order to take advantage of the increase in efficiency that QDs can provide, it is necessary to deposit the QDs into a medium that will not attenuate the incoming light and decrease efficiency. Future sections will discuss other materials that can be used as carrying mediums without the power loss caused by the PDMS.

\section{Chapter 4 - Discussion}

\subsection{Intensity and Decay of QDs with Time}

As previously discussed QDs are much more stable when shelled with ZnS. As described in section 3.4, it is important to ensure that the QDs will continue to remain stable over time due to the lifetime of the applications that they are paired with. Figure 4 indicates that the QDs remain stable over a three month period.

It is not uncommon for solar module manufacturers to offer a warranty that guarantees electrical production of $90 \%$ for 10 years and $80 \%$ for 25 years [23]. This means that solar cells that are paired with QDs must maintain $80 \%$ and $90 \%$ electrical output for 10 and 25 years respectively. For this reason the QDs paired with the solar cell must maintain the intensity they are initially rated at for 25 years. The test done in this work ensures that the QDs will continue to fluoresce at their initial intensity for three months but 
an additional test is necessary to disclose if the QDs exhibit any degree of intensity degradation over the course of 10 and 25 years as this will affect the electrical power output possible by the solar cell.

\subsection{Spectrometer Variation and Indications of Intensity}

As described in section 3.3, the design of the spectrometer causes it to have some variation in the data reported. In order to quantify the extent of the variation, the same sample of QDs was measured by the spectrometer during the same day while keeping the UV light at the same intensity. The same sample was measured and between each measurement the spectrometer was reset, the software restarted and the UV light was power cycled and bought to the same intensity. This was done to simulate the actions that are typically performed between measurements especially if the interval between measurements is days. Figure 11 and Table VII demonstrate the typical variation expected from the spectrometer.

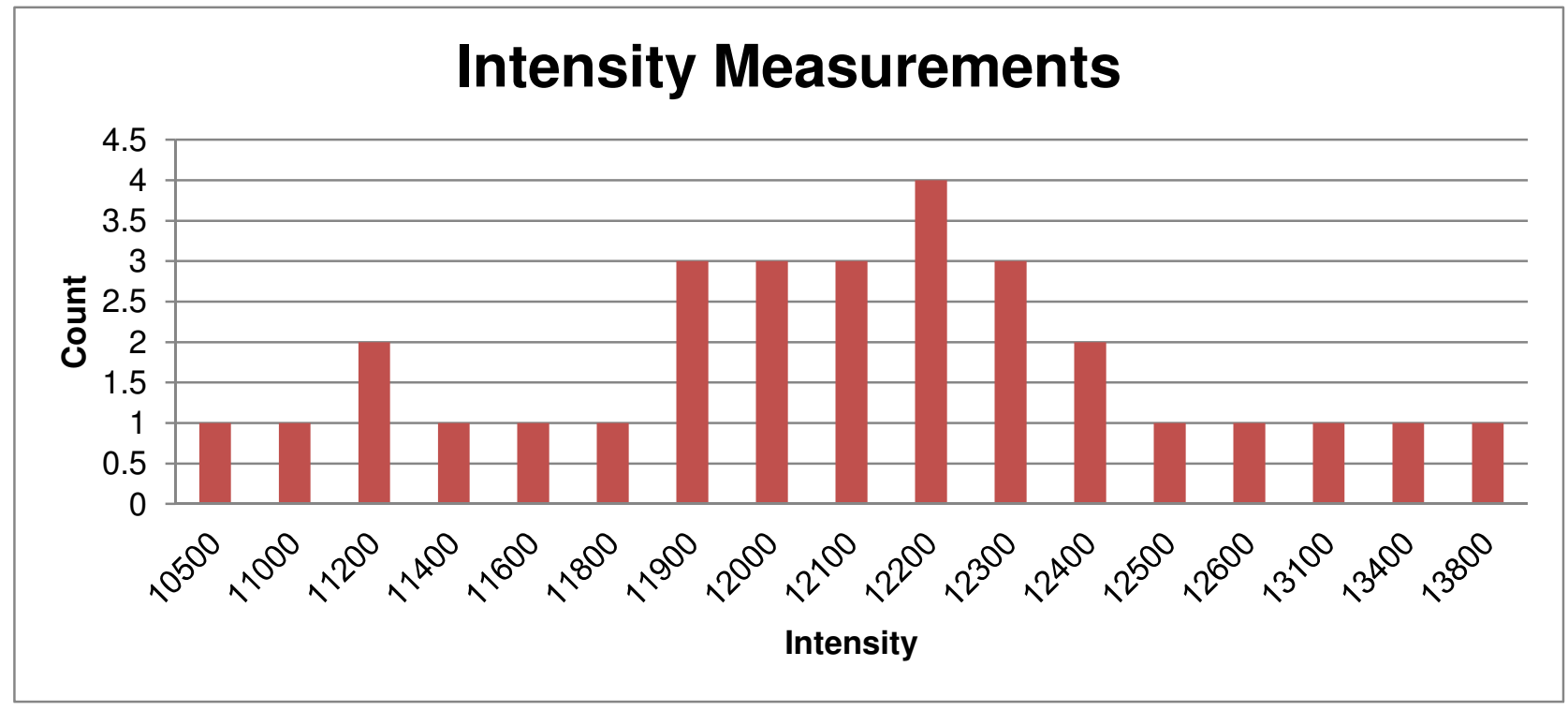

Figure 12: Intensity of the Same Sample Repeatedly Measured on a Single Day. 
Although the data does not appear to be a normal distribution, there are noticeably greater volumes of counts surrounding the mean which is 12,105 as shown in Table VII.

Table VII: Collected Intensity Measurements

\begin{tabular}{|c|c|c|}
\hline \multicolumn{2}{|l|}{ Intensity } & Count \\
\hline & 10500 & 1 \\
\hline & 11000 & 1 \\
\hline & 11200 & 2 \\
\hline & 11400 & 1 \\
\hline & 11600 & 1 \\
\hline & 11800 & 1 \\
\hline & 11900 & 3 \\
\hline & 12000 & 3 \\
\hline & 12100 & 3 \\
\hline & 12200 & 4 \\
\hline & 12300 & 3 \\
\hline & 12400 & 2 \\
\hline & 12500 & 1 \\
\hline & 12600 & 1 \\
\hline & 13100 & 1 \\
\hline & 13400 & 1 \\
\hline & 13800 & 1 \\
\hline MEAN & 12100 & \\
\hline RANGE & 3300 & \\
\hline STDEV & 851.08 & \\
\hline
\end{tabular}

This data is important for the user of the spectrometer so proper data collection can be done. When collecting intensity data on a sample, the user should collect the intensity 
on the sample multiple times and take the mean to ensure that the result is accurate. In order to ensure that the variation in metrology is not the cause of the variation in between the different data sets a sign test is performed to prove that there is a significant statistical significance with resulting $p$-values of less than 0.001 . The intensity data collected for this work was collected six times for each individual measurement, to reduce the amount of variation in the results. This fluctuation seen in Figure 4 can be attributed to this variation and Figure 6, 8 and 10 would possibly be made a lot more linear if this fluctuation was removed.

All intensity data was collected by taking the maximum of the intensity peaks for each measurement taken. The maximum of the intensity peak does not reveal the full nature of the QDs in question since there are other small peaks at different intensities that will cause a portion of the QDs to fluoresce at a different wavelength. This will cause inconsistencies when applying these QDs to a solar cell since not all the photons will fluoresce at the required wavelength.

The width of the intensity peak also indicates that the QDs being measured have a range of sizes and will fluoresce photons within the width of the peak as can be seen in Figure 13. The QDs that were applied to the solar cell were selected so that they have only one narrow peak in an attempt to eliminate this problem as much as possible. Although these precautionary measures were taken in the selection of the QDs, it's impossible to synthesize a batch of QDs that are all the same size. For this reason it may be necessary to develop a method to quantify the QDs that are within the width of the peak and those that form separate peaks as this will affect the performance of the solar cell that the QDs are applied to. It is also necessary to develop a method to synthesize the QDs so that they 
all fall within one narrow peak to minimize the issue associated with QDs fluorescing at a wavelength further away from the wavelength associated with the band gap of the solar cell.

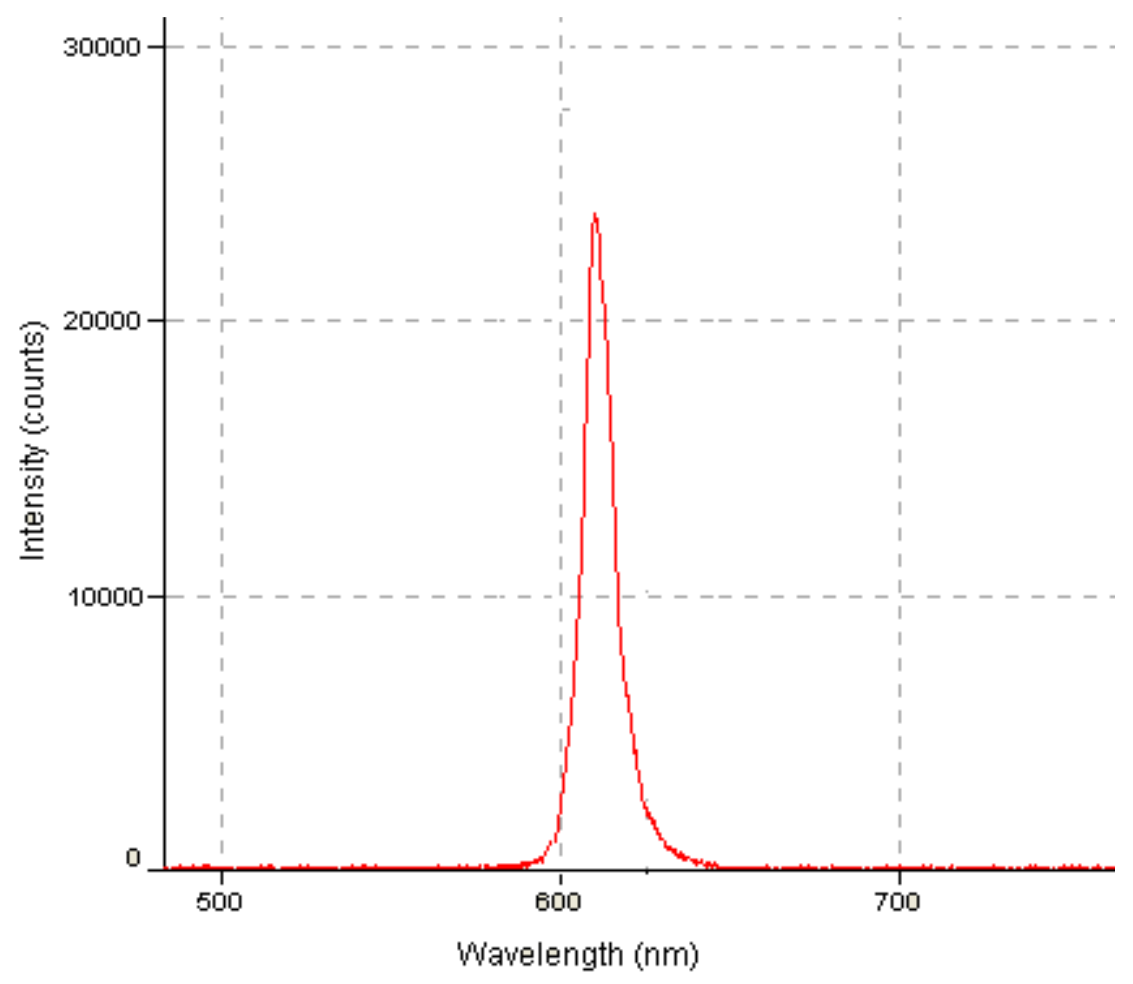

\section{Figure 13: Intensity Distribution of QDs}

The full width half $\max (\mathrm{FWHM})$ is $\sim 16 \mathrm{~nm}$ and the center wavelength $(\mathrm{CWL})$ is $\sim 615 \mathrm{~nm}$.

\subsection{Accuracy of the Intensity with Concentration Relation}

In order to ensure that the intensity with concentration relationship is accurate and repeatable it is necessary to ensure that the extracted and dried QDs are not contaminated with any other substance as this will decrease the intensity produced by the QDs and cause inconsistencies in the relationship. The QDs must be completely dried before they are weighed as the moisture will skew the data by increasing the mass. Once the QDs are 
extracted and dried, they are visibly inspected to confirm that they are completely dried. Since the dryness of the QDs is not quantified, fluctuations in the intensity with mass plot could be attributed to the additional moisture which causes QDs that appear to have a greater mass to have lower intensity.

Additional solids in solution that do not make up QDs including ZnS debris, Cd or Se that did not form QDs will cause the intensity for the given mass of the sample of QDs to appear lower. In order to minimize the error that could be caused by this issue, it is important to develop a process with well defined parameters that allows for the highest yield of QDs. An additional trial that would allow one to determine the yield of the synthesized QDs would be to determine the intensity of a given concentration of QDs with maximum yield and use the given intensity as a benchmark for future synthesis. In this way, one could determine the yield of QDs produced and this data could be used in conjunction with concentration data to determine expected intensity.

As discussed in the section 3.3, the thickness of the $\mathrm{ZnS}$ shell is directly related to quantum yield. A greater $\mathrm{ZnS}$ shell thickness results in greater quantum yield. The parameter that determines the thickness of the $\mathrm{ZnS}$ shell during the synthesis process is unknown so a metrology method to determine this thickness is necessary. The ZnS shell thickness could be determined using XRD, HRTEM and Raman. Although HRTEM alone will give a high resolution image of the QD, the interface between the core and the shell is not discernible so XRD and Raman need to be used as a complement to reveal more complete details of the structure [31]. Once a system of metrology is set up, data mining can be performed as the synthesis process is repeated to refine the process by which the ZnS shell is created and allow for the consistent synthesis of QDs with greater 
thicknesses.

\subsection{Loss of QD Intensity Upon Deposition in PDMS}

As discussed in section 3.3, there is a $\sim 2.75 \%$ decrease in the maximum power output of the solar cell when it is applied with PDMS. This can be due to the transmittance of PDMS and the reflection losses due to change in the refractive index caused by the addition of a medium. Although PDMS is very transparent, it has a light transmittance of approximately $95 \%$ [26]. Since this light transmittance is less than $100 \%$, the $\sim 2.75 \%$ decrease in maximum power output can be attributed to the light that is not transmitted through the PDMS and fails to reach the solar cell. In order to eliminate this problem the QDs need be deposited into a medium that has a greater transmittance. As discussed in section 1.3, spin on glass can be a good alternative to PDMS but every material will have some transmittance loss so it might be best to manufacture the panels with the QDs already incorporated in them.

Due to the existing transmittance loss associated with PDMS, the thickness of the PDMS layer applied to the solar cell will vary the transmittance. The thickness of the QD + PDMS layer applied to the solar cell was not optimized. Due to the difficulty of handling very thin layers of cured PDMS, a layer of PDMS no thinner than $1 \mathrm{~cm}$ thick was used in the solar cell application in this work. A thinner layer of PDMS would allow for greater transmittance but this must be done without compromising the QD concentration in the PDMS. Further work is necessary to optimize the thickness of the PDMS layer while maintaining the same QD concentration. This can possibly allow for greater power output if 
the same concentration can be maintained while reducing the transmittance losses.

\subsection{Solar Cell Power Output}

The solar cell power output is very dependent on the QD concentration, yield of the synthesized QDs in the batch and the distribution of the QDs in the PDMS. The distribution of the QDs in the PDMS can be maximized with proper agitation of the mixture until it appears completely uniform prior to curing so emphasis is on the first two elements. Due to the variation of the intensity that occurs over the same concentration, it is very important to incorporate the yield of the synthesized QDs. A high concentration does not necessarily indicate a high fluorescing intensity therefore a process that consistently yields QDs at maximum yield needs to be developed. Other solids in the QD mixture not only decrease the fluorescing intensity but also shield incoming light from reaching the solar cell and cause a further decrease in intensity.

The proper QD size is very significant as QDs that fluoresce in a region further away from the band gap will reduce the increase of efficiency exhibited. As indicated in section 3.3, the QDs that produced the result in this work fluoresce in the orange region but the amorphous silicon solar cell has a band gap with an associated wavelength slightly above the red region. Idealistically this panel would need to be applied with QDs that fluoresce in the lower infrared region, at $\sim 730 \mathrm{~nm}$. Optimization in QD synthesis is necessary to allow for the production of QDs that fluoresce at a narrow band directly surrounding the desired wavelength. Although the majority of the QDs utilized in this work 
fluoresce in the orange region, the small portion of QDs that fluoresce at wavelength in yellow and green regions further compromise the potential power gain.

\section{Chapter 5 - Conclusion}

\subsection{QD Stability}

As discussed in section 4.1, before one can consider combining QD technology with any other technology it is necessary to ensure that the QDs will perform their expected function for the duration of the lifetime of the product they are combined with. Solar cells are a good indicator of this, since they have an expected life of 20-25 years so the QDs must be able to fluoresce at a similar intensity during the lifetime of the panel, otherwise the panel will exhibit a decrease in power due to aging of the panel in addition to a decrease in power due to the failure of the QDs which will cause a larger net power loss in the panel.

The stability of the QDs is indicated by their intensity as measured by the spectrophotometer. QDs must be coated with ZnS in order to maintain their stability, and as discussed in section 4.3, the intensity of fluorescence of the QDs is also dependent on the thickness of the ZnS shell. There is an expected decay in the intensity of the QDs and this must be quantified over long periods to determine the expected efficiency loss in the application that the QDs are paired with.

\section{2 QD Metrology Indications}

Metrology is the only indication attainable on the quantum yield or concentration of the QDs. Due to the importance of this data, the methodology used to attain this data must 
allow for minimal error. As discussed in section 3.3, the QDs must be extracted from an octadecene solution, dried, weighed, and than redissolved in toluene to attain a concentration that can be measured on the spectrophotometer. Due to the multiple steps involved in this process care must be taken at each step to minimize the possible error.

The synthesis of the QDs does not guarantee the solution will be made up entirely of QDs. The intensity with concentration will vary depending on the amount of solid impurities present in solution. Solid impurities will lead to greater mass which will increase the concentration without an increase in intensity causing variations in the relationship between intensity and concentration. The yield of the QDs could be determined by the associated intensity, however, it can be difficult to determine the degree of relation associated with either the concentration or the QD yield. For this reason it can be very facilitative to utilize and alternate metrology method to capture QD yield.

QDs with higher yield will allow for a greater fluorescence which when deposited on the panel will result in greater efficiency. There is concentration saturation associated with the carrying medium in which the QDs are deposited, therefore lower intensity QDs will not yield the desired fluorescence before the medium is saturated. For this reason, QDs of higher yield are of substantial importance to achieve the maximum efficiency possible by the panel.

\subsection{Solar Cell Efficiency when Applied with QDs}

When a solar cell is irradiated with photons that are at or near the wavelength corresponding to the band gap of the panel, less of the higher energy photons excite electrons with more energy than the energy required to cross the band gap. The excess energy of these electrons is converted to heat which leads to a decrease in the panel 
efficiency. For this reason the application of QDs can lead to increase in power output. As indicated by the data presented in section 3.5 and 4.4 there is an increase in the power output of a solar cell when it is applied with QDs but there is also an attenuation associated with the application of PDMS due to light transmittance losses. The combination of both of these phenomenon results in a slight loss in the power output of the solar cell. A comparison of the power output of a solar cell with the application of only PDMS and that of a solar cell with PDMS + QDs indicates that there is a $\sim 2.5 \%$ increase in power output when the solar cell is applied with QDs.

The solar cell that was used for testing has a band gap of $1.7 \mathrm{eV}$ which corresponds to a wavelength of $\sim 730 \mathrm{~nm}$. Ideally the panel would be deposited with QDs that fluoresce at the wavelength corresponding to the band gap to achieve maximum efficiency but the QDs used fluoresced in the orange region or at about $620 \mathrm{~nm}$ which compromises the potential power increase possible. In order to maximize the power output possible by the application of the QDs it is also necessary to apply the panel with QDs of maximum yield. Although the QDs used in this work exhibited an approximate yield of $90 \%$, any loss in yield leads to a loss in the potential power output of the panel. The power output of the solar cell has the potential to increase when it is applied with QDs that fluoresce at the wavelength corresponding to the band gap and if the applied QDs exhibit the maximum possible yield.

\subsection{Attenuation due to PDMS}

As described in section 4.4, there is a measured attenuation of light in PDMS. As discussed, this is caused by the lack of full of transmittance in PDMS. PDMS has a 
transmittance of approximately $95 \%$, which is good for many applications but can pose problems for the solar cell. Although less than $5 \%$ of the light is prevented from reaching the solar cell, this is a significant loss when the QDs also provide a gain of less than $5 \%$. Although PDMS has qualities that make it a very lucrative carrying medium such as its low price, malleability, and short production time, it causes a prohibitive power loss. This work proves that there is a definite loss of power associated with the PDMS which compromises the power gain achieved by the QDs. Due to the power loss caused by the PDMS, it is necessary to investigate alternate carrying mediums with less attenuation or the application of QDs directly to the surface of the solar cell. Alternate materials as carrying mediums will be discussed in later sections.

\section{Chapter 6 - Future Work}

\subsection{Alternate Materials as Carrying Mediums}

As discussed in previous sections, PDMS causes a net power loss to the solar cell due to its transmittance. Since it is much more favorable from a manufacturing standpoint to deposit the QDs into a medium and subsequently apply this material onto the surface of the panel, alternate materials as carrying mediums should be considered first. The alternate material must be transparent with a transmittance as close to $100 \%$ as possible, malleable to allow for easy application to the panel, and less costly than the purchase of additional solar cell area or more costly higher efficiency solar cells.

Spin on Glass (SOG) is a carrying medium that should be explored since it can be spun onto a surface, cured and applied onto a solar cell. SOG must undergo high temperatures, in excess of $400{ }^{\circ} \mathrm{C}$ in order to cure. This poses a problem since such high 
temperature can lead to the permanent destruction of the QDs. If further research can lead to the synthesis of QDs that can withstand much higher temperature or the QDs can be deposited in the SOG without the susceptibility of destruction then SOG glass can be a possible carrying material. Most glasses have a transmittance of around $90 \%$ which is less than that of PDMS, however glass with much greater light transmission is also manufactured. The SOG needs to be made up of high transmission glass, which can have a transmission of up to $99 \%$ [25]. Such a high transmission will greatly minimize the attenuation of light associated with PDMS and allow for much greater power output from the solar cell.

Due to the difficulty in finding materials with high light transmission that can be spun on a surface, further research is necessary to find such materials in which the QDs can be deposited. In order to take the most advantage of the application of the QDs, the carrying medium needs to have a light transmission of $100 \%$. In order to ensure that no light is prevented from reaching the surface of the panel, it may be worthwhile to investigate applying the QDs directly on the surface of the panel. Since the QDs would no longer be deposited into a medium before they are applied to the surface of the panel, investigation of means to adhere the QDs to the surface is necessary. In addition the QDs will need to be applied uniformly; therefore a process to distribute QDs on the surface of the panel prior to adhering them is essential.

\subsection{Synthesis of Appropriate QDs}

As discussed in section 4.5, the QDs applied on the solar cell need to fluoresce at or near the band gap of the solar cell to take advantage of the increase in efficiency possible by irradiating a panel with photons that have a wavelength with the associated 
energy of the band gap. The steps of the current process utilized to synthesize QDs are not well quantified, which leads to inconsistent production of QDs. Further trials need to be performed to streamline steps of this process and allow for production of QDs with the desired size. Multiple synthesis operations may need to be performed while slightly modifying the parameters followed by data mining in order to find relationships between different parameters and the size of the resulting QDs. Once the parameters that determine size are well defined, QDs fluorescing in the red region or any region desired can be synthesized consistently. This will lead to QDs synthesis within a narrow wavelength ensuring that the QDs applied on the solar cell are optimized for its band gap.

\subsection{Solar Cells with Different Band Gaps}

These QDs were only applied on a single amorphous silicon solar cell with a band gap of $1.7 \mathrm{ev}$. Additional trials are required to prove if the application of QDs on solar cells with different band gaps will lead to a notable increase in efficiency. Since QDs can be synthesized to fluoresce in the visible and infrared region, different sized QDs can be applied to solar cells of different band gaps. The increase in power output may vary depending on the solar cell and greater power increases may be possible with combinations of different size QDs and band gaps. As mentioned in the previous section, different sized QDs can be produced much more consistently once the parameters that control the size of the QDs are well defined. It is possible that solar panels with band gaps associated with wavelengths in the infrared region would exhibit a greater power gain once applied with QDs since the wavelength associated with their band gap is much farther from the UV region. The application of QDs that fluoresce in the infrared region on solar cells 
that have a band gap with an associated wavelength in the infrared region would more drastically decrease the amount of heat produced and would allow for a greater power gain. An evaluation of the power output of different solar cells will lead to the disclosure of the solar cell that exhibits the most power gain. Discovery of the solar cell that leads to the most power output will allow for the production of the best QD and solar cell combination.

\subsection{Repeatability and Production}

Before the application of QDs can be put in production the synthesis of the QDs

must be well understood. QD synthesis at the desired wavelength and with an offset of no greater than $10 \mathrm{~nm}$ is needed in order to maximize the gain in power output. After well controlled synthesis of QDs is possible, the maximum gain in power output can be much more achievable. A concentration of QDs whether in solution or directly deposited on the solar cell must not be so high that QDs are receiving a reduced intensity of light due to obstruction from the neighboring QDs or so low that the effect of the QDs is minimized. The optimum concentration of QDs must be established before QD application on solar cells can be repeated in any volume. The established concentration of QDs must be well distributed to allow for the optimum irradiation of the desired photons on all areas of the solar cell. After parameters are well defined and power output is such that the investment is worthwhile, mass production of the solar cells applied with QDs can be made possible. 


\section{References}

1. William Shockley, Hans J Queisser,. Journal of Applied Physics (Palo Alto, 1961), Vol. 32, p. 510.

2. M. Cahay, Quantum Confinement VI : Nanostructured Materials and Devices. (The Electrochemical Society, Pennington, 2001), p. 33-34

3. Hartmut Haung; Stephan W. Koch, Quantum Theory of the Optical and Electronic Properties of Semiconductors. (World Scientific Publishing, Danvers, 2004), p. 383

4. Gildardo Delgado, Investigation of quantum confinement in silicon and germanium semiconductor nanocrystals and their application in photonic devices, Ph. D. thesis, Harvard University, 1997.

5. D.J. Norris, Measurement and assignment of the size-dependent optical spectrum in CdSe quantum dots, Ph.D. thesis Massachusetts Institute of Technology, 1995.

6. David F. Salisbury, Daniel Dubois.

http://news.vanderbilt.edu/2005/10/quantum-dots-that-produce-white-light-could-be-thelight-bulbs-successor-59204/

7. M. A.Walling; Novak, Shepard, Int. J. Mol. Sci. (Bethesda, 2009), Vol. 10, 441-491.

8. Neil Gershenfeld, Isaac L. Chuang, http://www.mat.ucm.es/catedramdeguzman/old/01historias/haciaelfuturo/Burgos09090 0/quantumcomputingSciAmer/0698gershenfeld.html

9. C.B. Murray, C.R. Kagan, M. G. Bawendi Annual Review of Materials Research (Yorktown, 2000), Vol. 30, p 545-610.

10. M. Nirmal, L. Brus, American Chemical Society (New York, 1998), Vol. 32, p. 407 - 414 
11. David Talbot (2007) "Nanocharging Solar" Technology Review. http://www.technologyreview.com/article/407470/tr10-nanocharging-solar/

12. Josh Angell, Synthesis and Characterization of CdSe-ZnS Core-Shell Quantum Dots for Increased Quantum Yield, M.S. thesis California Polytechnic University, SLO, 2011.

13. Henk Vrijhof,, ECETOC (Brussels, 1994) Vol. 20 Is. 55.

14. C Lötters, W Olthuis, P H Veltink and P Bergveld. Journal of Micromechanics and Microengineering (Bristol, 1997), Vol 7, p. 145.

15. Jessamine Ng Lee, Cheolmin Park, and George M. Whitesides. American Chemical Society (Washington D.C, 2003) Vol. 75, p. $6544-6554$.

16. E. Smulders, W. Rybinski, E. Sung, W. Rähse, J. Steber, F. Wiebel, A. Nordskog, http://onlinelibrary.wiley.com/doi/10.1002/14356007.a08_315.pub3/full

17. E.S. Kawasaki, A. Player, Nanomedicine: Nanotechnology Biology and Medicine. (Gaithersburg, 2005), Vol. 1, p. $101-109$.

18. Gao et al. Current opinion in biotechnology. (Atlanta, 2005) Vol. 16, p. 63-72

19. Alivisatos, et al. Annu. Rev. Biomed. Eng. (2005); Vol. 7, p. 55-76.

20. Chan et al. Current opinion in biotechnology. (2002) Vol. 13, p. 40-46

21. Michalet et al. Science. (2005), Vol. 307, p. 538-544.

22. M Grabolle, J. Ziegler, A. Merkulov, T. Nann, U. Resch-Genger. Ann N Y Acad Sci. (2008) Vol. 1130, p. 235-41

23. The SunPower Corporation. http://global.sunpowercorp.com/products/solarpanels/warranty/

24.Präzisions Glas \& Optik GmbH. Technical http://www.pgonline.com/int//katalog/curves/eagle2000_kurve.html 25. Water White Glass. http://www.waterwhiteglass.com/ 
26. David A. Chang-Yen, Richard K. Eich, and Bruce K. Gale. Journal of Light wave Technology. (2005), Vol. 23, Issue 6, p. 2088-2093.

27. leong Wong, Chih Ming Ho. Microfluid Nanofluidics. (2009), Vol. 7, p. $291-306$.

28. L. C. Sparks. Development and Characterization of Phospholipid Encapsulated Quantum Dot Constructs for Biologic Applications, M.S. thesis California Polytechnic University, SLO, 2012

29. A. Karczemska, A. Sokolowska. Journal of Wide Bandgap Materials (Lodz, 2012), Vol. 9 , p. $243-259$.

30. Francois Briquet, Andre Colas, Xavier Thomas. http://www.dowcorning.com/content/publishedlit/MMV0396-01.pdf

31. R.D. Lovchik, H. Wolf, E. Delamarche. Biomed Microdevices. (2011) Vol. 13, p. 1027 1032.

32. A. V. Baranov, Yu. P. Rakovich, J. F. Donegan. Physical Review B (2003) Vol. 68, p. $68-69$ 